\title{
Influence of higher $d$-wave gap harmonics on the dynamical magnetic susceptibility of high-temperature superconductors
}

\author{
A. P. Schnyder, A. Bill, and C. Mudry \\ Paul Scherrer Institute, CH-5232 Villigen PSI, Switzerland \\ R. Gilardi, H. M. Rønnow, and J. Mesot \\ Laboratory for Neutron Scattering, ETH Zurich and PSI Villigen, CH-5232 Villigen PSI, Switzerland \\ (Received 7 May 2004; revised manuscript received 6 July 2004; published 10 December 2004)
}

\begin{abstract}
Using a fermiology approach to the computation of the magnetic susceptibility measured by neutron scattering in hole-doped high- $T_{c}$ superconductors, we estimate the effects on the incommensurate peaks caused by higher $d$-wave harmonics of the superconducting order parameter induced by underdoping. The input parameters for the Fermi surface and $d$-wave gap are taken directly from angle-resolved photoemission experiments on $\mathrm{Bi}_{2} \mathrm{Sr}_{2} \mathrm{CaCu}_{2} \mathrm{O}_{8+x}$ (Bi2212). We find that higher $d$-wave harmonics lower the momentum-dependent spin gap at the incommensurate peaks as measured by the lowest spectral edge of the imaginary part in the frequency dependence of the magnetic susceptibility of Bi2212. This effect is robust whenever the fermiology approach captures the physics of high- $T_{c}$ superconductors. At energies above the resonance we observe diagonal incommensurate peaks. We show that the crossover from parallel incommensuration below the resonance energy to diagonal incommensuration above it is connected to the values and the degeneracies of the minima of the two-particle energy continuum.
\end{abstract}

DOI: 10.1103/PhysRevB.70.214511

PACS number(s): 71.30.+h, 72.15.Rn, 64.60.Fr, 05.40.-a

\section{INTRODUCTION}

The imaginary part $\chi^{\prime \prime}(\omega, \mathbf{q})$ of the magnetic susceptibility $\chi(\omega, \mathbf{q})$ probed by neutron scattering in high-temperature (high- $T_{c}$ ) superconductors is characterized by a very rich dependence on energy and momentum transfer, temperature, and doping. ${ }^{1-36}$ At temperatures well below the superconducting transition temperature $T_{c}$ and for a fixed frequency $\omega$ (of the order of the $d$-wave gap maximum around optimal doping) within a finite range of frequencies, $\chi^{\prime \prime}$ displays peaks at some symmetry related wave vectors in the Brillouin zone of the square lattice formed by planar $\mathrm{Cu}$ sites. The positions, heights, and widths of these peaks are temperature dependent and, in particular, are sensitive to the destruction of superconductivity above $T_{c}$ to a degree that depends on doping. As the energy transferred from the neutrons to the sample is varied, the positions in the Brillouin zone of the peaks in $\chi^{\prime \prime}$ also vary (as well as their heights and widths), i.e., the peaks are dispersing. The detailed intensity distribution of the dynamical spin susceptibility depends on the high- $T_{c}$ superconducting family.

For the $\mathrm{YBa}_{2} \mathrm{Cu}_{3} \mathrm{O}_{6+x}(\mathrm{YBCO})$ compounds it is observed that the separation in $\mathbf{q}$ space between four incommensurate peaks at the symmetry related wave vectors $\mathbf{q}=(\pi \pm \delta, \pi)$ and $\mathbf{q}=(\pi, \pi \pm \delta)$ decreases with increasing energy merging into a single resonance peak at the antiferromagnetic wave vector $(\pi, \pi)$ and at an energy of $41 \mathrm{meV}$ for optimally doped samples. ${ }^{1-19}$ Cooling below the superconducting transition temperature opens up a doping-dependent spin gap, which is proportional to $T_{c}{ }^{15}$

In the $\mathrm{La}_{2-x} \mathrm{Sr}_{x} \mathrm{CuO}_{4}$ (LSCO) family the dynamical magnetic susceptibility follows a similar dispersion. At energies above a spin gap four incommensurate peaks on the horizontal and vertical lines passing through $(\pi, \pi)$ in the Brillouin zone appear and disperse towards the antiferromagnetic zone center as the energy transfer is increased ${ }^{20-31}$ Although the incommensurate peaks in LSCO seem to join at the commensurate wave vector $\mathbf{q}=(\pi, \pi)$, no resonance peak has been observed in this compound to this date. Another remarkable observation in LSCO is that a spin gap has not been observed in the extreme underdoped and overdoped regimes. ${ }^{29,30}$

On $\mathrm{Bi}_{2} \mathrm{Sr}_{2} \mathrm{CaCu}_{2} \mathrm{O}_{8+x}$ (Bi2212) and $\mathrm{Tl}_{2} \mathrm{Ba}_{2} \mathrm{CuO}_{6+x}$ (TBCO) samples only a few inelastic neutron scattering measurements have been performed so far. For both compounds a resonance peak was observed, ${ }^{32-36}$ but an incommensurate signal below the resonance has not yet been measured, perhaps due to the limited size of single crystals currently available.

In this paper we will take the point of view that incommensurate and commensurate peaks of $\chi^{\prime \prime}$ have a common origin as they appear to be smoothly connected in the YBCO family. 9,11,12,15,37 This hypothesis seems hard to reconcile with theoretical scenarios based on the existence of dynamical stripes or on the $\mathrm{SO}(5)$ approach to high- $T_{c}$ superconductivity. In the dynamical stripe scenario incommensurate peaks are the natural descendants of the static charge and spin long-range order seen in $\mathrm{La}_{2-x} \mathrm{Ba}_{x} \mathrm{CuO}_{4}$ at $x=1 / 8,{ }^{38}$ say. ${ }^{39-45}$ In the $\mathrm{SO}(5)$ scenario a resonance at $(\pi, \pi)$ appears naturally as a result of an antibound state in the spin-triplet particle-particle channel. ${ }^{46-52}$ Commensurate and incommensurate peaks of $\chi^{\prime \prime}$ are smoothly connected in a scenario in which it is assumed that strongly renormalized quasiparticles close to the Fermi surface interact with a residual on-site Hubbard or nearest-neighbor antiferromagnetic interaction, in short a fermiology approach.

Common to all fermiology scenarios ${ }^{53-102}$ (see also Refs. 103-105 for related works on the "bare" magnetic susceptibility) is the random phase approximation (RPA) 


$$
\chi_{\mathrm{RPA}}(\omega, \mathbf{q})=\frac{g(\omega, \mathbf{q})}{1+h(\omega, \mathbf{q}) \Pi(\omega, \mathbf{q})}
$$

to the magnetic susceptibility. Here, $\Pi(\omega, \mathbf{q})$ is the ("bare") magnetic susceptibility of noninteracting fermionic (BCS) quasiparticles that depends sensitively on the Fermi surface (the superconducting BCS gap) above (below) $T_{c}$. The functions $g(\omega, \mathbf{q})$ and $h(\omega, \mathbf{q})$ are model dependent: A RPA treatment of the single-band Hubbard model with on-site repulsion $U$ yields $g(\omega, \mathbf{q})=\Pi(\omega, \mathbf{q})$ and $h(\omega, \mathbf{q})=-U .^{53-63} \mathrm{~A}$ RPA treatment of a single-band of fermionic (BCS) quasiparticles with a residual interaction such as a nearest-neighbor antiferromagnetic interaction of strength proportional to $J$ yields $g(\omega, \mathbf{q})=\Pi(\omega, \mathbf{q})$ and $h(\omega, \mathbf{q})=(J / 2)\left(\cos q_{x}+\cos q_{y}\right),{ }^{64-67}$ as is often done in the slave-boson treatment ${ }^{68-80}$ or the $1 / z$ expansion with $z$ the number of nearest neighbors of the $t$ - $J$ model. ${ }^{81-84}$ A RPA treatment on the bare static propagator $\chi_{0}(\mathbf{q})$ of collective spin-1 excitations due to a weak coupling as measured by the coupling constant $g$ with otherwise noninteracting fermionic (BCS) quasiparticles yields $g(\omega, \mathbf{q})$ $=\chi_{0}(\mathbf{q})$ and $h(\omega, \mathbf{q})=-g^{2} \chi_{0}(\mathbf{q}){ }^{85-89}$

The RPA approximation (1.1) has been improved in three ways. First, the single-band Hubbard model can be generalized to the three-band Hubbard model. ${ }^{90-94}$ Second, the feedback effect of the magnetic fluctuations encoded by Eq. (1.1) on the propagator of the fermionic (BCS) quasiparticles can be included self-consistently through the so-called fluctuation exchange (FLEX) approximation. ${ }^{95-100}$ Third, all unaccounted for interactions among the fermionic (BCS) quasiparticles can be included in a phenomenological way by substituting in Eq. (1.1) $\omega$ by $\omega+i \Gamma$ with $\Gamma$ a positive function of $\omega$, temperature $T$, doping $x$, and high- $T_{c}$ superconducting family. ${ }^{101,102}$

The goal of this paper is to assess the effects on the incommensurate peaks of the magnetic susceptibility $\chi$ caused by the presence of a higher $d$-wave harmonic in the superconducting order parameter of underdoped Bi2212. Indeed, it is observed in several angle-resolved photoemission (ARPES) experiments on underdoped Bi2212 that there are deviations from a pure $d$-wave order parameter. ${ }^{106-109}$ These deviations result in a rounding of the superconducting gap in the vicinity of its nodes. ${ }^{110}$ It is observed that the gap slope at the node decreases with underoping. ${ }^{111-113}$ We interpret this deviation as the signature of a $d$-wave order parameter extending to next-nearest-neighbor bonds between planar $\mathrm{Cu}$ sites, i.e., they push closer to 0 the anisotropy ratio $v_{\Delta} / v_{F}$ between the slope of the superconducting gap $v_{\Delta}$ and the Fermi velocity $v_{F}$, which are tangent and perpendicular to the Fermi surface, respectively.

Below, we adopt a phenomenological fermiology scenario by which we compute the RPA susceptibility (1.1) assuming that $\Pi(\omega, \mathbf{q})$ is calculated with the BCS dispersion measured by ARPES while $g(\omega, \mathbf{q})=\Pi(\omega, \mathbf{q})$ and $h(\omega, \mathbf{q})=-U$. To simplify the matter and to isolate the effect of decreasing $v_{\Delta}(x) / v_{F}(x)$ with underdoping, i.e., decreasing $x$, we assume that residual lifetime effects are small and substitute

$$
\omega \rightarrow \omega+i \Gamma,
$$

where $\Gamma=1 \mathrm{meV}$ is of the order of the energy resolution of most experiments. The lowest spectral edge in $\chi_{\mathrm{RPA}}^{\prime \prime}(\omega, \mathbf{q})$ considered as a function of $\omega$ with $\mathbf{q}$ held fixed defines the q-dependent spin gap. We find that increasing the higher harmonics by an increment of $15 \%$ relative to optimal doping, which corresponds to a decrease of $T_{c}$ by $28 \%$ in $\mathrm{Bi} 2212$ as was observed in Ref. 107, decreases the q-dependent spin gap by an amount that depends on the wave vector (see Figs. 3 and 4) and agrees qualitatively with YBCO measurements. This result is a robust feature of all fermiology scenarios and is consistent with the experimental observation of a fast decreasing (or even vanishing) spin gap with underdoping. 15,29

The paper is organized as follows. The fermiology approach is defined through a RPA magnetic susceptibility in Sec. II. Numerical results are presented in Sec. III and interpreted in Sec. IV. We close with a summary in Sec. V.

\section{RPA MAGNETIC SUSCEPTIBILITY}

One lesson inferred from ARPES data is that the lowenergy excitations in the superconducting state of Bi2212 are sharply defined quasiparticles obeying the BCS dispersion ${ }^{114}$

$$
E_{\mathbf{k}}=\sqrt{\varepsilon_{\mathbf{k}}^{2}+\Delta_{\mathbf{k}}^{2}}
$$

in the close vicinity to the Fermi surface defined by the condition $\varepsilon_{\mathbf{k}}=0$ and with a superconducting gap consistent with a $d$-wave symmetry, i.e.,

$$
\begin{gathered}
\Delta_{\mathbf{k}}=-\Delta_{\mathbf{k}^{\prime}},\left(k_{x}^{\prime}, k_{y}^{\prime}\right)=\left( \pm k_{y}, \mp k_{x}\right), \\
\Delta_{\mathbf{k}}=\Delta_{\mathbf{k}^{\prime}},\left(k_{x}^{\prime}, k_{y}^{\prime}\right)=\left( \pm k_{x}, \mp k_{y}\right) .
\end{gathered}
$$

This fact suggests that it might be plausible to treat the superconducting state of high- $T_{c}$ superconductors as a conventional BCS superconductor in the close vicinity to the Fermi surface, an assumption that we will make from now on. Following Norman in Ref. 62 the measured Fermi surface is fitted from the tight-binding expansion

$$
\varepsilon_{\mathbf{k}}=\frac{1}{2} \sum_{j=0}^{\infty} t_{j}\left(\cos \mathbf{a}_{j} \cdot \mathbf{k}+\cos \mathbf{b}_{j} \cdot \mathbf{k}\right)
$$

with $\mathbf{a}_{0}=\mathbf{b}_{0}=0$ and $\mathbf{a}_{j}, \mathbf{b}_{j}$ a pair of orthogonal vectors joining a site of the square lattice to two of its $j$ th nearest neighbors. Similarly, the $d$-wave BCS gap is fitted from the tightbinding expansion

$$
\begin{aligned}
\Delta_{\mathbf{k}}= & \Delta_{1}\left(\cos k_{x}-\cos k_{y}\right)+\Delta_{2}\left(\cos 2 k_{x}-\cos 2 k_{y}\right) \\
& +\Delta_{3}\left(\cos 2 k_{x} \cos k_{y}-\cos k_{x} \cos 2 k_{y}\right)+\cdots .
\end{aligned}
$$

In practice we truncate the expansions at $j=5$ for the Fermi surface and to the first two terms for the $d$-wave BCS gap. As opposed to the slave-boson approach which attempts to capture the doping dependence of the $t_{j}$ 's and the $\Delta_{j}$ 's from the large $U$ limit of the Hubbard model, we will take the phenomenological point of view that the BCS dispersion (2.1) of the "bare" quasiparticles is an input deduced from ARPES data for the Bi2212 family.

We will assume in this paper that anomalous features at energy scales far away from the Fermi energy, say of the order $2 \max _{\mathbf{k}} \Delta_{\mathbf{k}}$ such as is the case for the incommensurate 
and commensurate peaks observed at optimal doping with inelastic neutron scattering, can be accounted for by postulating the existence of "bare" noninteracting BCS quasiparticles whose dispersion is given by a fit to ARPES data and which interact weakly through some small residual interactions which we take to be an on-site Hubbard repulsion with coupling constant $U$. In this spirit, the renormalization of the "bare" magnetic susceptibility

$$
\begin{gathered}
\Pi(\omega, \mathbf{q})=\frac{1}{N} \sum_{\mathbf{k}} \sum_{s^{\prime}, s= \pm} \frac{C_{\mathbf{q}, \mathbf{k}}^{s^{\prime}, s}\left[f\left(s^{\prime} E_{\mathbf{k}+\mathbf{q}}\right)-f\left(s E_{\mathbf{k}}\right)\right]}{\sigma\left(\omega+i 0^{+}\right)-\left(s^{\prime} E_{\mathbf{k}+\mathbf{q}}-s E_{\mathbf{k}}\right)}, \\
\sigma=- \text { if } s^{\prime}=s=-1,+ \text { otherwise, } \\
C_{\mathbf{q}, \mathbf{k}}^{s^{\prime}, s}=\frac{1}{4}\left(1+s^{\prime} s \frac{\varepsilon_{\mathbf{k}+\mathbf{q}} \varepsilon_{\mathbf{k}}+\Delta_{\mathbf{k}+\mathbf{q}} \Delta_{\mathbf{k}}}{E_{\mathbf{k}+\mathbf{q}} E_{\mathbf{k}}}\right),
\end{gathered}
$$

is, within the RPA,

$$
\chi_{\mathrm{RPA}}(\omega, \mathbf{q})=\frac{\Pi(\omega, \mathbf{q})}{1-U \Pi(\omega, \mathbf{q})} .
$$

Here, $N$ is the number of sites on a square lattice with periodic boundary conditions, $f(x)=[\exp (\beta x)+1]^{-1}$ is the Fermi distribution ( $\beta$ the inverse temperature), and the BCS dispersion (2.1) is used.

In Figs. 1(a) and 1(b) we present a plot of the static limit of the RPA magnetic susceptibility (2.2b) and of the static "bare" magnetic susceptibility (2.2a), respectively. It is seen that there are no significant differences between the static limits of the "bare" and RPA magnetic susceptibilities for small momentum transfer $\mathbf{q}$ (small forward scattering) but that for large momentum transfer, $\mathbf{q}$ close to $(\pi, \pi)$, the renormalization effects are of order 1 as they signal the proximity to an antiferromagnetic instability of the BCS ground state. These renormalization effects modify the quasiparticle self-energy and have been proposed as an explanation to the peak-dip-hump shape seen in the ARPES signal as a function of frequency. ${ }^{74,115}$ In this paper we do not consider this feedback on the quasiparticle self-energy. However, since the effects of higher $d$-wave harmonics on the RPA magnetic susceptibility are robust, we expect them to survive in a selfconsistent approach.

We close this section by noting that the imaginary part $\Pi^{\prime \prime}(\omega, \mathbf{q})$ of the "bare" susceptibility (2.2a) simplifies to

$$
\begin{gathered}
\Pi^{\prime \prime}(\omega, \mathbf{q})=\frac{\pi}{N} \sum_{\mathbf{k}} C_{\mathbf{q}, \mathbf{k}}^{+,-} \delta\left(\omega-E_{2}(\mathbf{q}, \mathbf{k})\right), \\
C_{\mathbf{q}, \mathbf{k}}^{+,-}=\frac{1}{4}\left(1-\frac{\varepsilon_{\mathbf{k}+\mathbf{q}} \varepsilon_{\mathbf{k}}+\Delta_{\mathbf{k}+\mathbf{q}} \Delta_{\mathbf{k}}}{E_{\mathbf{k}+\mathbf{q}} E_{\mathbf{k}}}\right), \\
E_{2}(\mathbf{q}, \mathbf{k})=E_{\mathbf{k}+\mathbf{q}}+E_{\mathbf{k}},
\end{gathered}
$$

in the zero-temperature limit and for positive frequencies as is relevant in inelastic neutron scattering. We shall see in Sec. IV that the numerical results from Sec. III can be understood in terms of the frequency dependence of the twoparticle density of states (DOS) a)

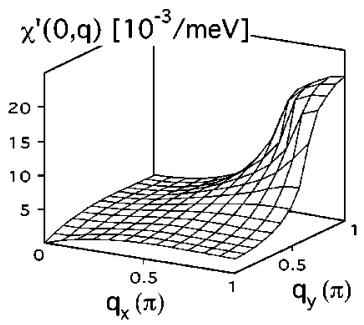

b)

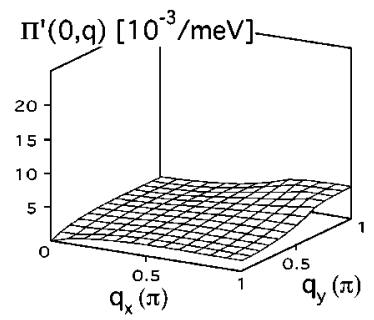

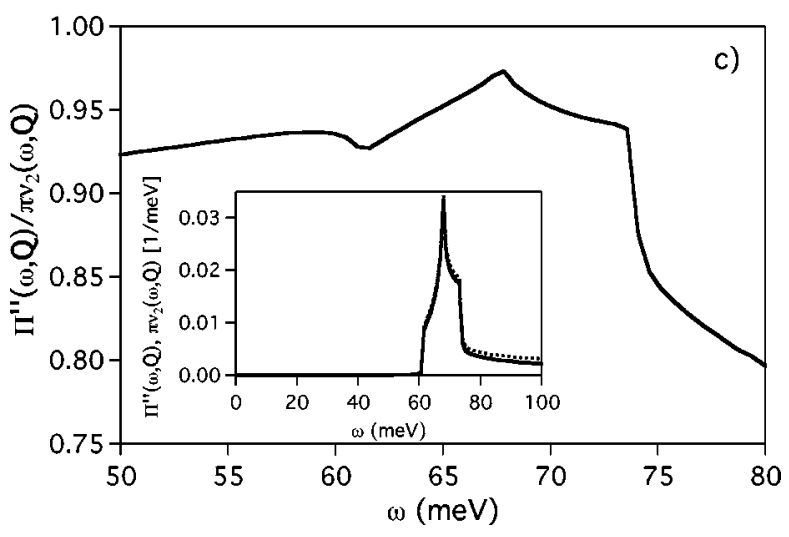

FIG. 1. Plot of the real parts $\chi_{\mathrm{RPA}}^{\prime}(0, \mathbf{q})$ (a) and $\Pi^{\prime}(0, \mathbf{q})$ (b) as a function of $\mathbf{q}$ in the upper-right quadrant of the Brillouin zone for the BCS dispersion of Bi2212 at optimal doping (see Table I). (c) Plot of $\Pi^{\prime \prime}(\omega, \pi, \pi) /\left[\pi \nu_{2}(\omega, \pi, \pi)\right]$ as a function of $\omega>0$ in the relevant energy range for the BCS dispersion of Bi2212 at optimal doping (see Table I). The inset displays the $\omega$ dependence of the imaginary part $\Pi^{\prime \prime}(\omega, \pi, \pi)$ (thick line) and $\pi \nu_{2}(\omega, \pi, \pi)$ (dashed line). The temperature is here taken to be $T=0 \mathrm{~K}$ while a damping $\Gamma=0.1 \mathrm{meV}$ is used.

$$
\nu_{2}(\omega, \mathbf{q}):=\frac{1}{2 N} \sum_{\mathbf{k}} \delta\left(\omega-E_{2}(\mathbf{q}, \mathbf{k})\right)
$$

since it differs very little from that of $\Pi^{\prime \prime}(\omega, \mathbf{q}) / \pi$ for a fixed wave vector $\mathbf{q}$ close to the commensurate vector $(\pi, \pi)$ and at very low temperatures, as is illustrated in Fig. 1(c).

\section{NUMERICAL RESULTS}

We have calculated numerically the RPA (2.2b) to the magnetic susceptibility with the inclusion of lifetime effects implemented through substitution (1.2). Hereby, we are using the BCS dispersion (2.1) with the hopping and superconducting parameters extracted from fits to the Fermi surface and gap measured with ARPES within the Bi2212 family for the underdoped and optimally doped regimes (see Refs. 62, 107, and 116-118). These parameters can be found in Table I. For each doping concentration the value of the on-site Hubbard repulsion $U$ is chosen so that the energy of the resonance is consistent with unpolarized neutron data in Refs. 32 and 34 and with the indirect determination of the resonance energy via the peak-dip-hump feature from APRES data in Refs. 119 and 120 . The value of the coupling constant $U$ increases with underdoping, a trend consistent with the naive expectation that moving away from half-filling in the Hubbard model 
TABLE I. Values of the parameters used to fit the superconducting gap (see Ref. 107) in the Bi2212 family measured with ARPES as a function of doping. The gap parameters for $T_{c}=87 \mathrm{~K}, T_{c}$ $=83 \mathrm{~K}$, and $T_{c}=75 \mathrm{~K}$ follow from a fit to ARPES data (Ref. 107), and for $T_{c}=68 \mathrm{~K}$ from an extrapolation. For the band parameters we use the same values as Norman in Ref. 62 (units in meV): $t_{0}$ $=87.9, t_{1}=-554.7, t_{2}=132.7, t_{3}=13.2, t_{4}=-184.9$, and $t_{5}=26.5$. The last row lists the values of the coupling constant $U$. The doping decreases when reading the columns from left to right: The second, third, fourth, and fifth columns correspond to optimally doped, slightly underdoped, and underdoped samples, respectively. All quantities are in units of meV.

\begin{tabular}{crrrr}
\hline \hline Parameters & $T_{c}=87 \mathrm{~K}$ & $T_{c}=83 \mathrm{~K}$ & $T_{c}=75 \mathrm{~K}$ & $T_{c}=68 \mathrm{~K}$ \\
\hline$\Delta_{1}$ & 18.3 & 18.8 & 18.8 & 19.6 \\
$\Delta_{2}$ & -2.1 & -3.9 & -5.8 & -8.8 \\
$\Delta_{0}$ & 35.0 & 37.0 & 38.0 & 41.0 \\
$B$ & 0.96 & 0.92 & 0.89 & 0.84 \\
$d \tilde{\Delta} /\left.d \phi\right|_{\phi=\pi / 4}$ & 57.0 & 52.0 & 44.8 & 36.3 \\
$U$ & 165 & 173 & 180 & 191 \\
\hline \hline
\end{tabular}

reduces the effect of the strong interactions. ARPES experiments on Bi2212 indicate that the Fermi surface is (if at all) only weakly dependent on doping. ${ }^{117,118}$ To simplify matters, we have implemented this observation by keeping the hopping parameters unchanged as a function of doping.

To perform the summations in formula (2.2b), we meshed the Brillouin zone with $1024 \times 1024$ points. In order to reduce the effects of a finite lifetime in Fig. 1, we reduced $\Gamma$ in Eq. (1.2) from the experimental resolution $\sim 1$ $\mathrm{meV}$ to $0.1 \mathrm{meV}$. The temperature is taken to be vanishing in most instances except in Figs. 3 and 6 where $T=5 \mathrm{~K}$.

It is convenient to use polar coordinates to represent the wave-vector dependence of the superconducting gap on the Fermi surface. To this end, define along the arc of the Fermi surface that belongs to the upper-right quadrant of the Brillouin zone the angle $0 \leqslant \phi \leqslant \pi / 2$ through [see Fig. 2(a)]

$$
\phi=\arctan \left(\frac{\pi-k_{y}}{\pi-k_{x}}\right) .
$$

In terms of this angle, define the auxiliary gap function

$$
\begin{gathered}
\widetilde{\Delta}(\phi):=\Delta_{0}[B \cos (2 \phi)+(1-B) \cos (6 \phi)], \\
\Delta_{0}:=\max _{\mathbf{k} \in \text { Fermi surface }} \Delta_{\mathbf{k}} .
\end{gathered}
$$

The auxiliary gap function $\widetilde{\Delta}(\phi)$ obeys the same $d$-wave symmetry as the gap function $\Delta_{\mathbf{k}}$ does on the Fermi surface. The parameter $B$ entering $\widetilde{\Delta}(\phi)$ is taken to be a function of doping $x$ that can be determined for Bi2212 from ARPES measurements (see Table I). ${ }^{107}$ The agreement between $\widetilde{\Delta}(\phi)$ and $\Delta_{\mathbf{k}}$ for $\mathbf{k}$ on the Fermi surface is excellent. The slope of the superconducting gap $v_{\Delta}$ tangent to the Fermi surface at the node of the gap is proportional to $(d \tilde{\Delta}) /(d \phi)$ at $\phi=\pi / 4$. It decreases with underdoping as is apparent from Table I.
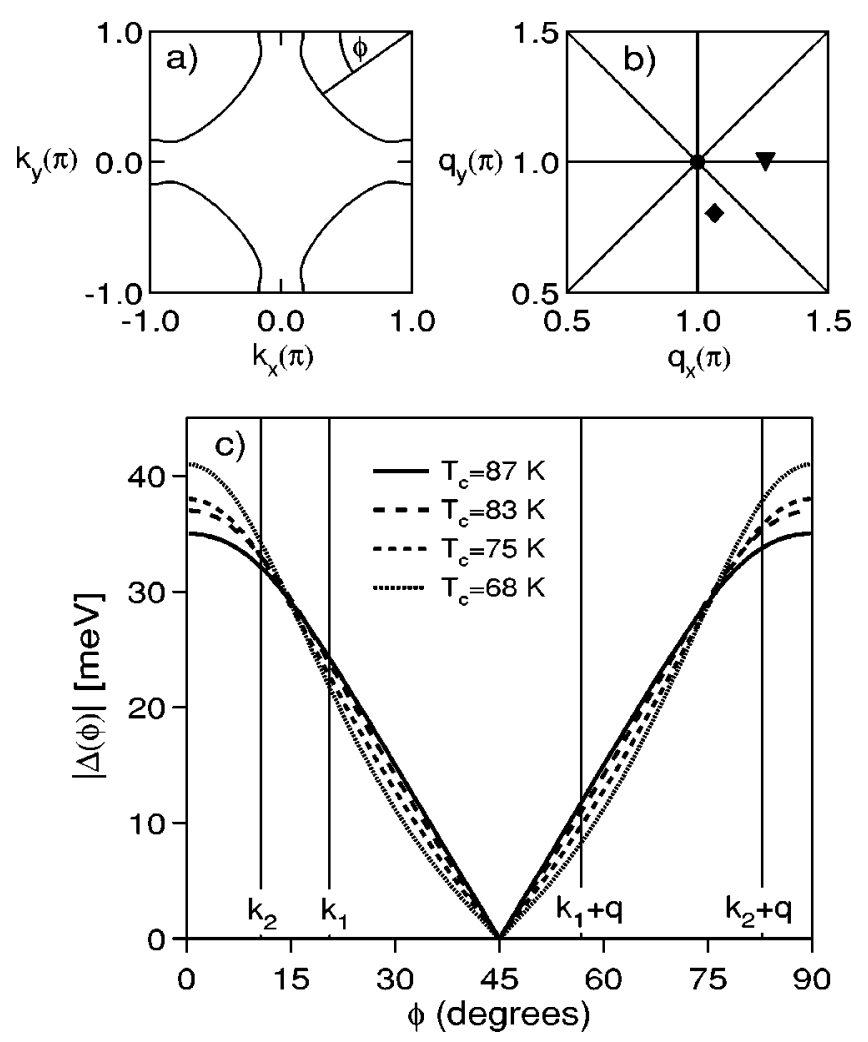

FIG. 2. Panel (a) displays the Fermi surface of Bi2212 with $t_{0}, \ldots, t_{5}$ as in Table I. The angle $\phi$ measures the position along the Fermi arc centered about $(\pi, \pi)$ (holelike) in the Brillouin zone. Panel (b) displays the reciprocal q space together with the symmetry axes (parallel and diagonal) and the three wave vectors $(\pi, \pi)$, $(1.26 \pi, \pi)$, and $(1.065 \pi, 0.805 \pi)$. Panel (c) displays the absolute value of the gap function $|\Delta(\phi)|$ on the Fermi surface as a function of Fermi surface angle $\phi$ for Bi2212 with the parameters of Table I. Plotted are the gaps for optimally doped samples (solid line), slightly underdoped (long dashed line), and underdoped samples (dashed and dotted lines). For the momentum transfer $\mathbf{q}$ $=(1.26 \pi, \pi)$ vertical lines at the Fermi surface angles, where the pairwise nested wave vectors $\mathbf{k}_{1}, \mathbf{k}_{1}+\mathbf{q}, \mathbf{k}_{2}$ and $\mathbf{k}_{2}+\mathbf{q}$ lie, are drawn (see Sec. IV).

The gap function $\Delta_{\mathbf{k}}$ on the Fermi surface is depicted in Fig. 2(c) for the parameters of Table I.

In Figs. 3(a)-3(c) we illustrate for three different wave vectors depicted in Fig. 2(b), $\mathbf{q}=(\pi, \pi)$, the antiferromagnetic wave vector, $\mathbf{q}=(1.26 \pi, \pi)$, a wave vector on the horizontal symmetry axis passing through $(\pi, \pi)$, and $\mathbf{q}=(1.065 \pi, 0.805 \pi)$, a wave vector off the symmetry axes, how the dependences on frequency of the imaginary part $\chi_{\mathrm{RPA}}^{\prime \prime}(\omega+i \Gamma, \mathbf{q})$ of the RPA magnetic susceptibility $(2.2 \mathrm{~b})$ changes with underdoping. In each panel the four curves correspond to the four dopings in Table I. As the doping decreases (i.e., decreasing $T_{c}$ ) the leading edge moves to the left (i.e., to lower frequencies). The q-dependent spin gap, which we define by the lowest spectral edge in $\chi_{\mathrm{RPA}}^{\prime \prime}$ considered as a function of frequency, decreases with decreasing doping for both the commensurate wave vector $(\pi, \pi)$ and the incommensurate points $(1.26 \pi, \pi)$ and $(1.065 \pi, 0.805 \pi)$ on the symmetry axis and off the symmetry axis passing 


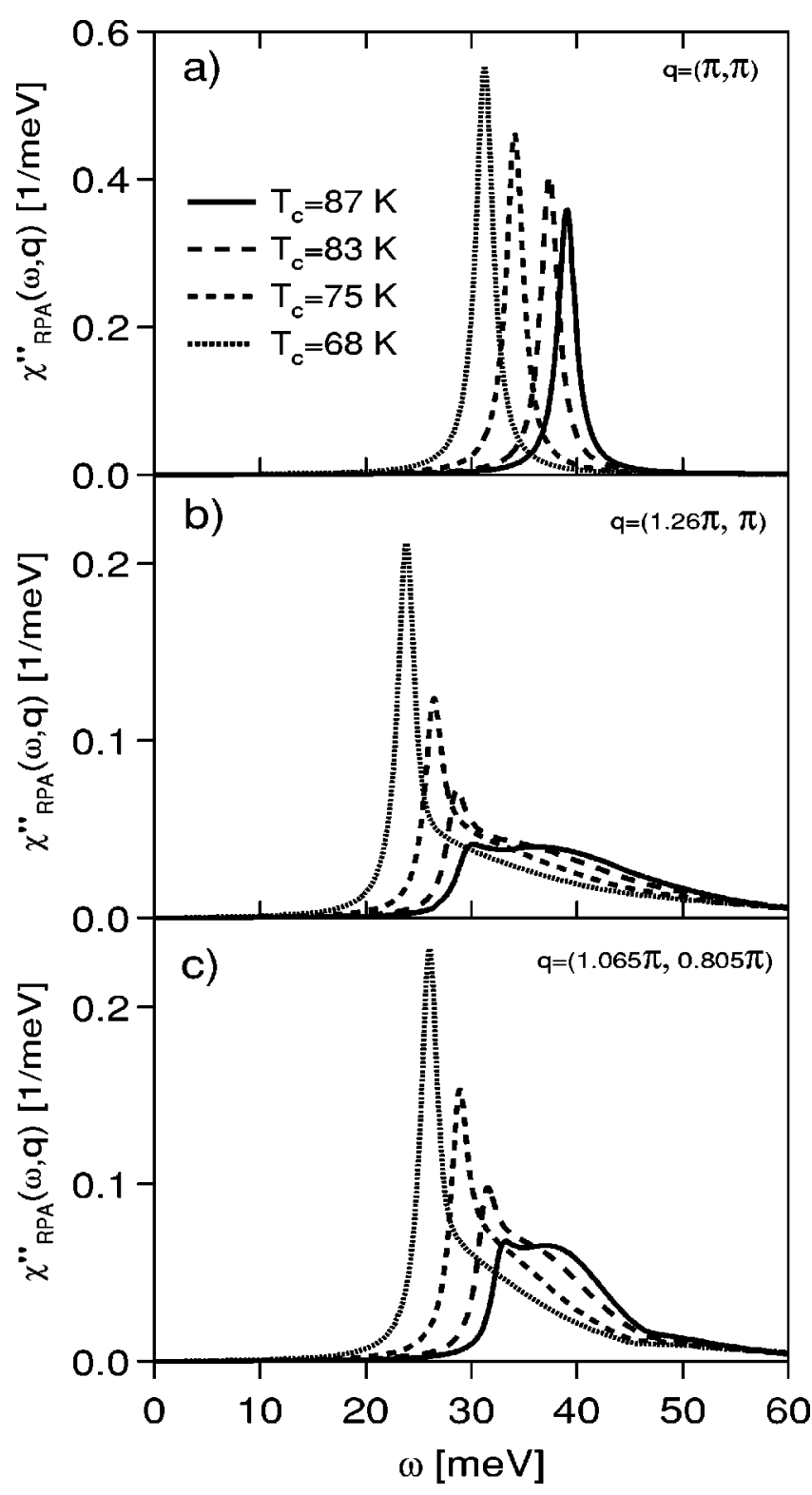

FIG. 3. Panel (a) displays the frequency dependences of $\chi_{\mathrm{RPA}}^{\prime \prime}(\omega, \mathbf{q})$ at the commensurate wave vector $\mathbf{q}=(\pi, \pi)$ for four different values $x$ of doping in the Bi2212 family with the BCS dispersions taken from Table I. Curves with the leading edge moving to the left (i.e., to lower frequencies) have decreasing doping $x\left(T_{c}\right)$. Panels (b) and (c) are the same as panel (a) except for the incommensurate wave vectors $\mathbf{q}=(1.26 \pi, \pi)$ and $\mathbf{q}$ $=(1.065 \pi, 0.805 \pi)$ being held fixed. For all three wave vectors the q-dependent spin gap decreases with underdoping. The temperature is here taken to be $T=5 \mathrm{~K}$ while a damping $\Gamma=1 \mathrm{meV}$ is used.

through $(\pi, \pi)$, respectively. This behavior is consistent with the observed doping dependence of the q-dependent spin gap in the LSCO and YBCO families. The intensities of the peaks in all panels of Figs. 3(a)-3(c) increase with underdoping. This increase in intensity is accompanied by a narrowing of the line shape in panels $b$ and $c$.

In Fig. 4 the intensities of the imaginary part of the RPA spin susceptibility are shown as a function of frequency and wave vector for the four doping concentrations of Table I. At

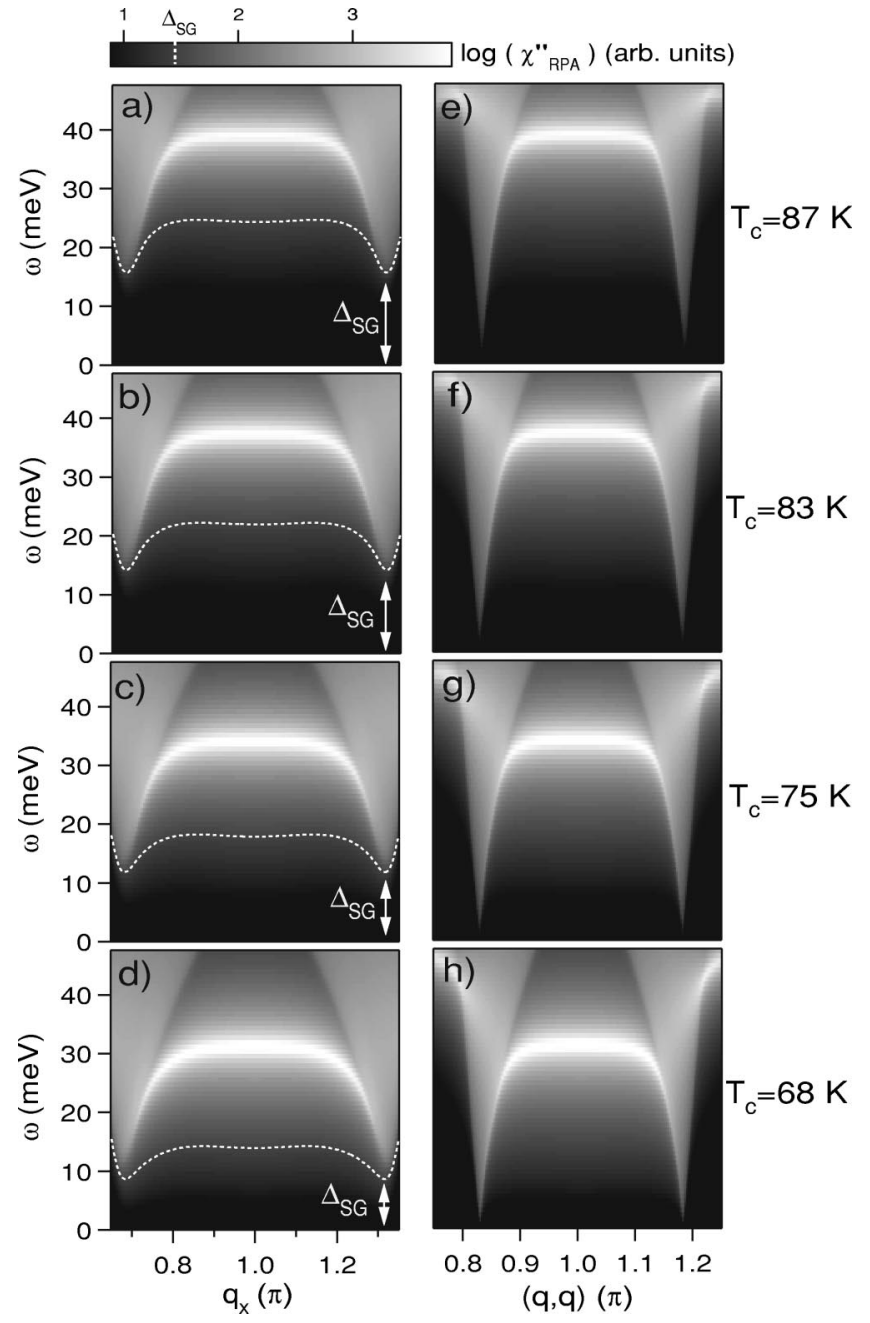

FIG. 4. Panels (a)-(d) display the intensities of $\chi_{\mathrm{RPA}}^{\prime \prime}\left(\omega, q_{x}, \pi\right)$ in $\log _{10}$ scale in the $\left(\omega, q_{x}\right)$ plane at fixed $q_{y}=\pi$ for four different doping concentrations in the Bi2212 family with the BCS dispersions taken from Table I. Panels (a), (b), (c), and (d) correspond to samples with a transition temperature $T_{c}$ of $87,83,75$, and $68 \mathrm{~K}$, respectively. As a guide to the eye contour lines are drawn at an intensity which is about $0.5 \%$ of the maximum intensity of the dynamical spin susceptibility. Panels (e)-(h) are the same as panels (a)-(d) except for the $\mathbf{q}$ vector which runs along the diagonal instead of the horizontal line in reciprocal space. The temperature is here taken to be $T=0 \mathrm{~K}$ while a damping $\Gamma=1 \mathrm{meV}$ is used.

the corresponding resonance energies (39, 37, 34, and $31 \mathrm{meV}$, respectively) the peaks are at the $(\pi, \pi)$ point. When the frequency is reduced from the resonance energy, dominant incommensurate peaks in the $(\pi \pm \delta, \pi)$ and $(\pi, \pi \pm \delta)$ direction occur, and a subdominant structure on the diagonal lines which pass through two incommensurate points in $\mathbf{q}$ space shows up. The peaks are dispersing with a downward curvature. Above the resonance energy the dominant peaks cross over from the parallel to the diagonal symmetry axes passing through $(\pi, \pi)$. The same happens below $19.2 \mathrm{meV}\left(T_{c}=87 \mathrm{~K}\right), 17.6 \mathrm{meV}\left(T_{c}=83 \mathrm{~K}\right), 15.2 \mathrm{meV}\left(T_{c}\right.$ $=75 \mathrm{~K}$ ), and $12.8 \mathrm{meV}\left(T_{c}=68 \mathrm{~K}\right)$, respectively. (We will then speak of parallel and diagonal peaks, respectively.) For frequencies larger than the resonance energy incommensu- 
rate peaks on the diagonal symmetry axes passing through the antiferromagnetic wave vector $(\pi, \pi)$ have recently been observed in both $\mathrm{La}_{2-x} \mathrm{Ba}_{x} \mathrm{CuO}_{4}$ (Ref. 38) and YBCO. ${ }^{19} \mathrm{Ex}-$ perimentally this diagonal pattern is seen up to $200 \mathrm{meV}$ in $\mathrm{La}_{2-x} \mathrm{Ba}_{x} \mathrm{CuO}_{4}$, and up to $100 \mathrm{meV}$ in YBCO, i.e., well beyond the regime of applicability of our model (say $2 \max _{\mathbf{k}} \Delta_{\mathbf{k}}$ away from the Fermi energy). The crossover to dominant diagonal peaks at low energies has not been observed in experiments. If the wave vector is restricted to the horizontal symmetry axes in the Brillouin zone, the spin gap $\Delta_{\mathrm{SG}}\left(q_{x}, \pi\right)$ remains finite for all values of $q_{x}$. However, if the wave vector lies on the diagonal symmetry axes, the spin gap $\Delta_{\mathrm{SG}}\left(q_{x}, q_{x}\right)$ vanishes for certain values of $q_{x}=q_{y}$.

The dispersion of the peak positions of $\chi_{\mathrm{RPA}}^{\prime \prime}$ for the four doping concentrations of Table I and with $\mathbf{q}$ moving along the horizontal or diagonal lines passing through $(\pi, \pi)$ are shown in Figs. 5(a) and 5(b), respectively. With underdoping the bell-like shape of the peak dispersion moves to lower energies implying a decrease of the q-dependent spin gap (see also Figs. 3 and 4). The degree of incommensuration measured by the separation $2 \delta$ of the two peaks of $\chi_{\mathrm{RPA}}^{\prime \prime}$ decreases as one moves from the optimally doped to the underdoped regimes. This agrees with neutron scattering measurements on the YBCO and LSCO families. ${ }^{15,27}$

\section{DISCUSSION}

In this section we review the mechanism causing commensurate resonance peaks and incommensurate peaks in the numerical simulations of the imaginary part of the RPA magnetic susceptibility. Up to multiplication with the BoseEinstein distribution, inelastic neutron scattering has direct access to the imaginary part $\chi^{\prime \prime}(\omega, \mathbf{q})$ of the magnetic susceptibility $\chi(\omega, \mathbf{q})$, which is given by

$$
\chi_{\mathrm{RPA}}^{\prime \prime}(\omega, \mathbf{q})=\frac{\Pi^{\prime \prime}(\omega, \mathbf{q})}{\left[1-U \Pi^{\prime}(\omega, \mathbf{q})\right]^{2}+\left[U \Pi^{\prime \prime}(\omega, \mathbf{q})\right]^{2}}
$$

within the RPA approximation $\left[\chi^{\prime}(\omega, \mathbf{q})\right.$ and $\Pi^{\prime}(\omega, \mathbf{q})$ denote the real parts of $\chi(\omega, \mathbf{q})$ and $\Pi(\omega, \mathbf{q})$, respectively]. The "bare" imaginary part $\Pi$ " $(\omega, \mathbf{q})$ of the magnetic susceptibility controls $\chi_{\mathrm{RPA}}^{\prime \prime}(\omega, \mathbf{q})$ in two ways. First, $\chi_{\mathrm{RPA}}^{\prime \prime}(\omega, \mathbf{q})$ vanishes whenever $\Pi^{\prime \prime}(\omega, \mathbf{q})$ does and $1 \neq U \Pi^{\prime}(\omega, \mathbf{q})$. Second, at q held fixed, any steplike discontinuity in the frequency dependence of $\Pi^{\prime \prime}(\omega, \mathbf{q})$ at some frequency $\omega_{n}(\mathbf{q})$ results in a logarithmic divergence of the frequency dependence of $\Pi^{\prime}(\omega, \mathbf{q})$ at $\omega_{n}(\mathbf{q})$ through the Kramers-Kronig relation between the imaginary and real parts of causal response functions. This in turn guarantees that (i) the dynamical Stoner condition

$$
1-U \Pi^{\prime}(\omega, \mathbf{q})=0
$$

can be met at the frequency

$$
\omega_{n}^{*}(\mathbf{q})<\omega_{n}(\mathbf{q})
$$

and (ii) $\chi_{\mathrm{RPA}}^{\prime \prime}(\omega, \mathbf{q})$ acquires a pole at $\omega_{1}^{*}(\mathbf{q})$ and peaks at $\omega_{\mathrm{n}}^{*}(\mathbf{q})$, where $n>1$ indexes the remaining jump discontinuities of $\Pi^{\prime \prime}(\omega, \mathbf{q})$.
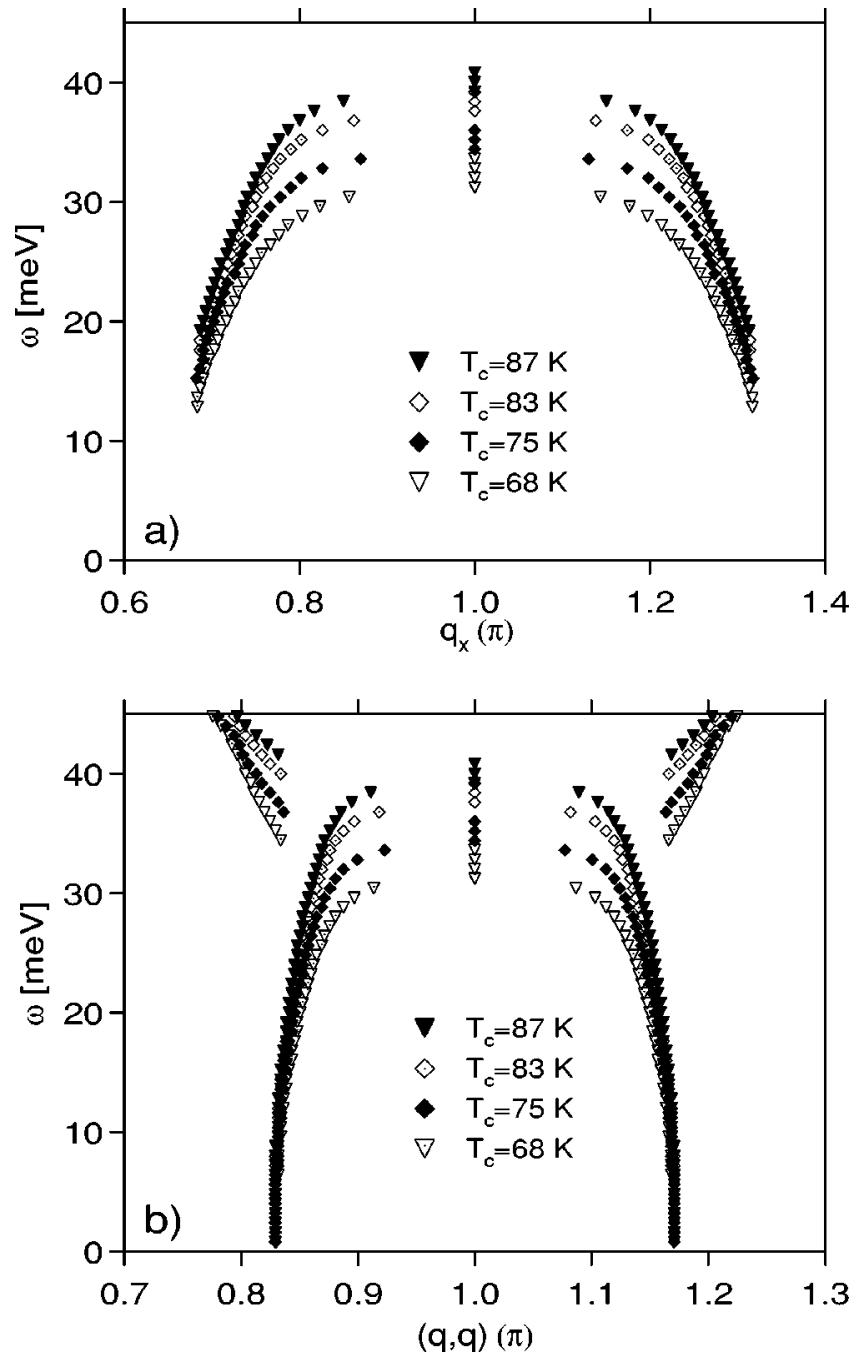

FIG. 5. (a) Peak positions of $\chi_{\mathrm{RPA}}^{\prime \prime}(\omega, \mathbf{q})$ for fixed $q_{y}=\pi$ as a function of $q_{x}$ and energy with the BCS dispersions taken from Table I for four different samples with a transition temperature $T_{c}$ of $87 \mathrm{~K}$ (solid triangles), $83 \mathrm{~K}$ (open diamonds), $75 \mathrm{~K}$ (solid diamonds), and $68 \mathrm{~K}$ (open triangles). (b) Peak positions of $\chi_{\mathrm{RPA}}^{\prime \prime}(\omega, \mathbf{q})$ as function of frequency and momentum transfer with $\mathbf{q}$ along the diagonal $q_{x}=q_{y}$ for the same four doping concentrations as in panel (a). Above the resonance the diagonal peaks are the dominant structure. The same happens at very low energies. At intermediate energies, the diagonal peaks are subdominant with the parallel peaks being dominant (see Fig. 4). The temperature is here taken to be $T=0 \mathrm{~K}$ while a damping $\Gamma=1 \mathrm{meV}$ is used.

For any small but finite damping $\Gamma, \Pi^{\prime \prime}(\omega+i \Gamma, \mathbf{q})$ becomes a nonvanishing and continuous function of $\omega>0$ when holding q fixed. Similarly, the logarithmic singularities in the $\omega$ dependences of $\Pi^{\prime}(\omega, \mathbf{q})$ are cut off by a finite $\Gamma$ under the substitution (1.2). Under this substitution the dynamical Stoner criterion (4.2a) can only be met for a sufficiently large size of the step in $\Pi^{\prime \prime}(\omega, \mathbf{q})$, and the pole at $\omega_{1}^{*}$ in $\chi_{\mathrm{RPA}}^{\prime \prime}(\omega, \mathbf{q})$ turns into a peak of finite height

$$
\chi_{\mathrm{RPA}}^{\prime \prime}\left(\omega_{1}^{*}+i \Gamma, \mathbf{q}\right)=\frac{1}{U^{2} \Pi^{\prime \prime}\left(\omega_{1}^{*}+i \Gamma, \mathbf{q}\right)}
$$

with the full width at half maximum (FWHM) 


$$
\mathrm{FWHM}=\left.\frac{2 \Pi^{\prime \prime}(\omega+i \Gamma, \mathbf{q})}{\partial \Pi^{\prime}(\omega+i \Gamma, \mathbf{q}) / \partial \omega}\right|_{\omega=\omega_{1}^{*}}
$$

To sum up, the condition for dynamical Stoner enhancement of $\chi_{\mathrm{RPA}}^{\prime \prime}(\omega, \mathbf{q})$, a collective effect, can be reduced to the condition for $\Pi^{\prime \prime}(\omega, \mathbf{q})$ to have steplike discontinuities as a function of frequency when holding the wave vector fixed.

In view of Eqs. (2.3) and (2.4) steplike discontinuities in the frequency dependence of $\Pi^{\prime \prime}(\omega, \mathbf{q})$ with $\mathbf{q}$ held fixed (at zero temperature and with the inverse lifetime $\Gamma$ infinitesimally small) are closely related to steplike discontinuities in the frequency dependence of the two-particle DOS (2.4) at fixed q. These jump discontinuities in $\nu_{2}(\omega, \mathbf{q})$ occur whenever the two-particle energy $E_{2}(\mathbf{q}, \mathbf{k})$ in Eq. (2.3) reaches a local minimum $\omega_{n}$ at the $\mathbf{q}$-dependent wave vector $\mathbf{k}_{n}$, in which case the size of the step is proportional to ${ }^{121}$

$$
\begin{gathered}
\left(m_{n}^{(1)} m_{n}^{(2)}\right)^{1 / 2}, \\
0<\frac{1}{m_{n}^{(1,2)}}=\left.\frac{\partial^{2} E_{2}(\mathbf{q}, \mathbf{k})}{\partial k_{1,2} \partial k_{1,2}}\right|_{\mathbf{k}=\mathbf{k}_{n}} .
\end{gathered}
$$

The steplike discontinuity in the two-particle DOS $\nu_{2}(\omega, \mathbf{q})$ at $\omega_{n}$ is present in $\Pi^{\prime \prime}(\omega, \mathbf{q})$ if and only if the coherence factor $C_{\mathbf{q}, \mathbf{k}}^{+,-}$from Eq. (2.3) is nonvanishing in a neighborhood of $\mathbf{k}_{n}$. The steplike jump is turned into a smooth increase otherwise. ${ }^{104}$

It is shown in the Appendix that, when $\mathbf{q}$ is chosen not to be on any of the diagonals of the Brillouin zone or the horizontal and vertical lines passing through the commensurate point $(\pi, \pi)$ [see Fig. 2(b)], there are four distinct but twofold degenerate local minima $\omega_{1}<\omega_{2}<\omega_{3}<\omega_{4}$ of $E_{2}(\mathbf{q}, \mathbf{k})$ for the parameters of Table I located at the $\mathbf{q}$-dependent wave vector $\mathbf{k}_{1}, \mathbf{k}_{2}, \mathbf{k}_{3}$, and $\mathbf{k}_{4}$, respectively, in the Brillouin zone. When $\mathbf{q}$ is chosen to be on the diagonals that pass through the commensurate point $(\pi, \pi)$, but away from it, there are three distinct local minima $\omega_{1}<\omega_{2}<\omega_{3}$ at $\mathbf{k}_{1}, \mathbf{k}_{2}$, and $\mathbf{k}_{3}$, respectively, which are either doubly degenerate $\left(\omega_{1}\right.$ and $\left.\omega_{3}\right)$ or four-fold degenerate $\left(\omega_{2}\right)$. If the momentum transfer lies on the horizontal or vertical lines passing through the commensurate point, but away from it, there are two distinct but four-fold degenerate local minima $\omega_{1}<\omega_{2}$ of $E_{2}(\mathbf{q}, \mathbf{k})$ located at the $\mathbf{q}$-dependent wave vector $\mathbf{k}_{1}$ and $\mathbf{k}_{2}$, respectively. Finally, all local minima collapse to one global but eight-fold degenerate minimum $\omega_{1}$ located at $\mathbf{k}_{1}$ when $\mathbf{q}=(\pi, \pi)$ or one of its symmetry-related images.

Two criteria control the size of the first step with increasing $\omega$ : (i) How large the effective masses $m_{1}^{(1,2)}$ are, i.e., how flat the two-particle dispersion is, (ii) how large the degeneracy of the global minimum is. It turns out that, when $\mathbf{q}$ is chosen to be at the commensurate antiferromagnetic vector $(\pi, \pi)$, the geometrical mean of the effective masses $m_{1}^{(1,2)}$ becomes very large because of the proximity of $\mathbf{k}_{1}$ to an extended saddle point for the parameters of Table I. Furthermore this effect is magnified by the eight-fold degeneracy of the global minimum of the two-particle dispersion at $\mathbf{q}$ $=(\pi, \pi)$. Taken together conditions (i) and (ii) ensure that the dynamical Stoner criterion is met at an energy

$$
\omega_{1}^{*} \equiv \omega_{\mathrm{AF}}^{*}
$$

sufficiently far below the two-particle continuum threshold for the resonant nature of the resulting spin-1 collective excitation not to be washed out by a finite temperature of $5 \mathrm{~K}$ or a damping of $1 \mathrm{meV}$. The eight-fold degeneracy is partially broken to a four-fold degeneracy when $\mathbf{q}$ moves away from $(\pi, \pi)$ along the horizontal or vertical lines passing through $(\pi, \pi)$ (parallel incommensuration) with $\mathbf{k}_{1}$ moving towards the nodal line and $\mathbf{k}_{2}$ moving away from it [see Fig. $2(c)]$. For energies below the commensurate resonance energy

$$
\omega_{1}^{*} \equiv \omega_{\| \mathrm{IC}}^{*}<\omega_{\mathrm{AF}}^{*},
$$

this turns the resonant commensurate peak into a downward dispersing incommensurate peak, which eventually becomes nonresonant due to the failure to meet the Stoner criterion. By energy conservation the dominant incommensurate peaks cross over to the diagonals of the Brillouin zone at frequencies much lower than $\omega_{\mathrm{AF}}^{*}$ since $\mathbf{q}$ then connects regions close to the nodes of the BCS dispersion. ${ }^{103}$ When the wave vector is pushed away from $(\pi, \pi)$ along the diagonals, the eight-fold global minimum splits into three distinct minima. The first minimum $\omega_{1}$ being lowest in energy leads to the diagonal peaks at low energies. Together with the lowest minimum of the two-particle energy for $\mathbf{q}$ off the symmetry axes it is also the cause of the subdominant structure on the diagonal lines passing through $(\pi+\delta, \pi)$ and $(\pi, \pi+\delta)$, say, at intermediate energies. The second four-fold degenerate minimum lies in general at energies just above the resonance $\omega_{\mathrm{AF}}^{*}$, but below the eight-fold degenerate minima at $\mathbf{q}=(\pi, \pi)$, and is thus responsible for the crossover to diagonal incommensurate peaks above the resonance energy. Finally, by choosing $\mathbf{q}$ away from the symmetry axes one spreads the spectral weight of the commensurate resonance among four distinct two-fold degenerate local minima, three of which are in the two-particle continuum, thereby loosing the most in height and sharpness of the peak relative to the line shape of the commensurate resonance as a function of frequency.

To conclude, the crossover from diagonal to parallel incommensuration with increasing frequency at low energies and from parallel to diagonal peaks with increasing frequency above the resonance energy is brought about by the onset of four-fold degenerate minima of the two-particle dispersion $E_{2}(\mathbf{q}, \mathbf{k})$ at the corresponding energies. This effect is quite sensitive to both the shape of the BCS dispersions and the details of the residual interaction among the BCS quasiparticles: On the one hand, an antiferromagnetic interaction with $h(\omega, \mathbf{q})=(J / 2)\left(\cos q_{x}+\cos q_{y}\right)$ leads to an enhancement of the diagonal peaks compared to the parallel ones, which potentially destroys the crossover to parallel incommensuration upon increasing frequency towards the resonance. ${ }^{62}$ On the other hand, it was observed in Refs. 76 and 78 that the parallel peaks remain dominant at energies below but not too far from the resonance in a slave-boson mean-field approach to the $t-t^{\prime}-J$ model.

At low energies the intensity of $\chi_{\mathrm{RPA}}^{\prime \prime}(\omega+i \Gamma, \mathbf{q})$ for a fixed wave vector dies off quickly (see Figs. 3 and 4). In the fol- 
lowing we shall define the lowest, sharp spectral edge in $\chi_{\mathrm{RPA}}^{\prime \prime}(\omega, \mathbf{q})$ considered as a function of frequency at a fixed wave vector $\mathbf{q}$ to be the $\mathbf{q}$-dependent spin gap. By Eqs. (2.3) and (4.1), the q-dependent spin gap for vanishing damping $\Gamma$ is given by the threshold to the two-particle continuum provided there is no resonant excitation below the threshold. Otherwise, the q-dependent spin gap is determined by the energy of the lowest bound state. Any sharp spectral edge in $\chi_{\mathrm{RPA}}^{\prime \prime}(\omega, \mathbf{q})$ turns into a smooth edge by the inclusion of finite lifetime effects through substitution (1.2) in which case, to a first approximation, the q-dependent spin gap is given by the position of the half maximum of the leading edge on the low frequency side.

The effects on the q-dependent spin gap caused by the change of the gap parameters in Table I induced by underdoping can be best understood by use of arguments based on pseudo-Fermi-nesting. A good approximation for the position of the q-dependent (local) minima of $E_{2}(\mathbf{q}, \mathbf{k})$ is obtained by requesting that both $\mathbf{k}_{n}$ and $\mathbf{k}_{n}+\mathbf{q}$ lie on the Fermi surface,

$$
0=\varepsilon_{\mathbf{k}_{n}}=\varepsilon_{\mathbf{k}_{n}+\mathbf{q}},
$$

and that the coherence factor $C_{\mathbf{q}, \mathbf{k}}^{+,-}$be maximal, i.e.,

$$
\operatorname{sgn}\left(\Delta_{\mathbf{k}_{n}} \Delta_{\mathbf{k}_{n}+\mathbf{q}}\right)=-1 .
$$

For a fixed wave vector $\mathbf{q}$ the steplike discontinuity of the frequency dependence of $\Pi^{\prime \prime}(\omega, \mathbf{q})$ is then approximately located at the energy

$$
\widetilde{\omega}_{n}=\left|\Delta\left(\widetilde{\mathbf{k}}_{n}\right)\right|+\left|\Delta\left(\widetilde{\mathbf{k}}_{n}+\mathbf{q}\right)\right|,
$$

where $\tilde{\mathbf{k}}_{n}$ are the solutions of the equation set (4.6a). In particular, $\widetilde{\omega}_{1}$ determines approximately the threshold to the two-particle continuum.

At frequencies much smaller than the resonance energy $\omega_{\mathrm{AF}}^{*}$ the approximate two-particle threshold $\widetilde{\omega}_{1}$ is effectively governed by excitations whose pseudo-Fermi-nesting vectors connect parts of the Fermi surface which are close to the nodes. Hence, at these energies the decreasing slope of the gap function $(d \Delta) /(d \phi)$ at $\phi=\pi / 4$ results in a decrease of the approximate two-particle threshold $\widetilde{\omega}_{1}$ [see vertical lines $\mathbf{k}_{1}$ and $\mathbf{k}_{1}+\mathbf{q}$ in Fig. 2(c)].

For frequencies which are comparable to the resonance energy however, the two-particle threshold increases with the change of the gap parameters induced by underdoping. This can be understood from the fact that at frequencies close to, or higher than the resonance energy the approximate twoparticle threshold $\widetilde{\omega}_{1}$ is controlled by excitations with pseudo-Fermi-nesting vectors that connect parts of the Fermi surface which are in the vicinity of $(0, \pi)$ or one of its symmetry related images. In these parts of the Fermi surface the increase of the gap maximum $\Delta_{0}$ causes the gap function to increase with underdoping [see vertical lines $\mathbf{k}_{2}$ and $\mathbf{k}_{2}+\mathbf{q}$ in Fig. 2(c)].

On top of the effects caused by the change of the gap parameters there is an additional modification of $\chi_{\mathrm{RPA}}^{\prime \prime}(\omega$ $+i \Gamma, \mathbf{q})$ with underdoping induced by the increase of the coupling constant $U$. Owing to the failure to meet the Stoner criterion, the frequency of the incommensurate peaks at a given wave vector $\mathbf{q}$ and at energies much smaller than the resonance energy is not changed by a not-too-large increase in $U$. Thus, in the limit of a $\Gamma=0$, the q-dependent spin gap is unaffected in this energy range by a not-too-large increase of the coupling constant. For energies close to the resonance energy $\omega_{\mathrm{AF}}^{*}$ though, the increase of $U$ with underdoping causes both the q-dependent spin gap and the frequency of the (in)commensurate peaks to decrease. The quantitative role played by $U$ can be illustrated by reproducing the frequency dependences of the RPA susceptibility in Fig. 3 with all BCS parameters unchanged but with $U$ fixed to its value at optimal doping. The result is displayed in Figs. 6(a) and 6(b) where one sees that (i) the resonance energy moves with underdoping to higher energies as a result of the increasing gap maximum in panel 6(a), and (ii) the leading edge moves with underdoping to lower energies for the wave vector $(1.26 \pi, \pi)$ in panel $6(\mathrm{~b})$. (The position of the leading edge is defined to be at the half maximum of the edge on the lowfrequency side.) We see that the spin gap value at a wave vector sufficiently far away from $(\pi, \pi)$ is rather insensitive to keeping $U$ constant as in Fig. 6(b) or to adjusting $U$ so as to get the correct resonance energy as in Fig. 3(b). We conclude that the dominant doping dependence of the q-dependent spin gap comes from the doping dependence of the higher harmonics for a wave vector sufficiently far away from $(\pi, \pi)$.

This insensitivity depends in a crucial way on how large $U$ becomes with underdoping. This can be illustrated in a rather dramatic way by switching off the BCS parameter $\Delta_{2}$ for all dopings. The parameter $\Delta_{1}$ is then chosen so that the maximum of the superconducting gap on the Fermi surface $\Delta_{0}$ agrees with the values in Ref. 107, i.e., we find that $\Delta_{1}$ equals $18.8,19.9,20.5$, and $22.1 \mathrm{meV}$, for samples with a $T_{c}$ of $87,83,75$, and $68 \mathrm{~K}$, respectively. For the coupling constant $U$ we demand that its value is chosen so as to reproduce the same resonances as in Fig. 3(a), i.e., we find that $U$ takes the values $170,184,196$, and $211 \mathrm{meV}$, when $T_{c}$ takes the values $87,83,75$, and $68 \mathrm{~K}$, respectively. Evidently, the increase of $U$ with underdoping is now much stronger than in Table I. Having recalibrated $U$ to the new parameters of the BCS dispersion, we plot in Fig. 6(c) the frequency dependence of $\chi^{\prime \prime}(\omega+i \Gamma, \mathbf{q})$ for the wave vector $(1.26 \pi, \pi)$. Comparison of Fig. 6(c) with Fig. 3(b) shows that a broad peak at optimal doping can be turned into a resonance with underdoping due to a too strong increase in $U$.

To summarize, we expect that the q-dependent spin gap always decreases with underdoping. Far away from the antiferromagnetic wave vector $(\pi, \pi)$ this is mostly a consequence of the decreasing slope of the gap function at the node, whereas close to $(\pi, \pi)$, it is a result of both the increasing coupling constant $U$ and the increasing higher harmonics. This is confirmed by the numerical simulations presented in Sec. III. The effect of a decreasing q-dependent spin gap upon changing the gap parameters induced by underdoping is present in all fermiology scenarios. For example, we have also performed calculations with a residual nearest-neighbor antiferromagnetic interaction instead of the on-site Hubbard repulsion as well as within a RPA treatment of the bare propagator of collective spin- 1 excitations weakly 


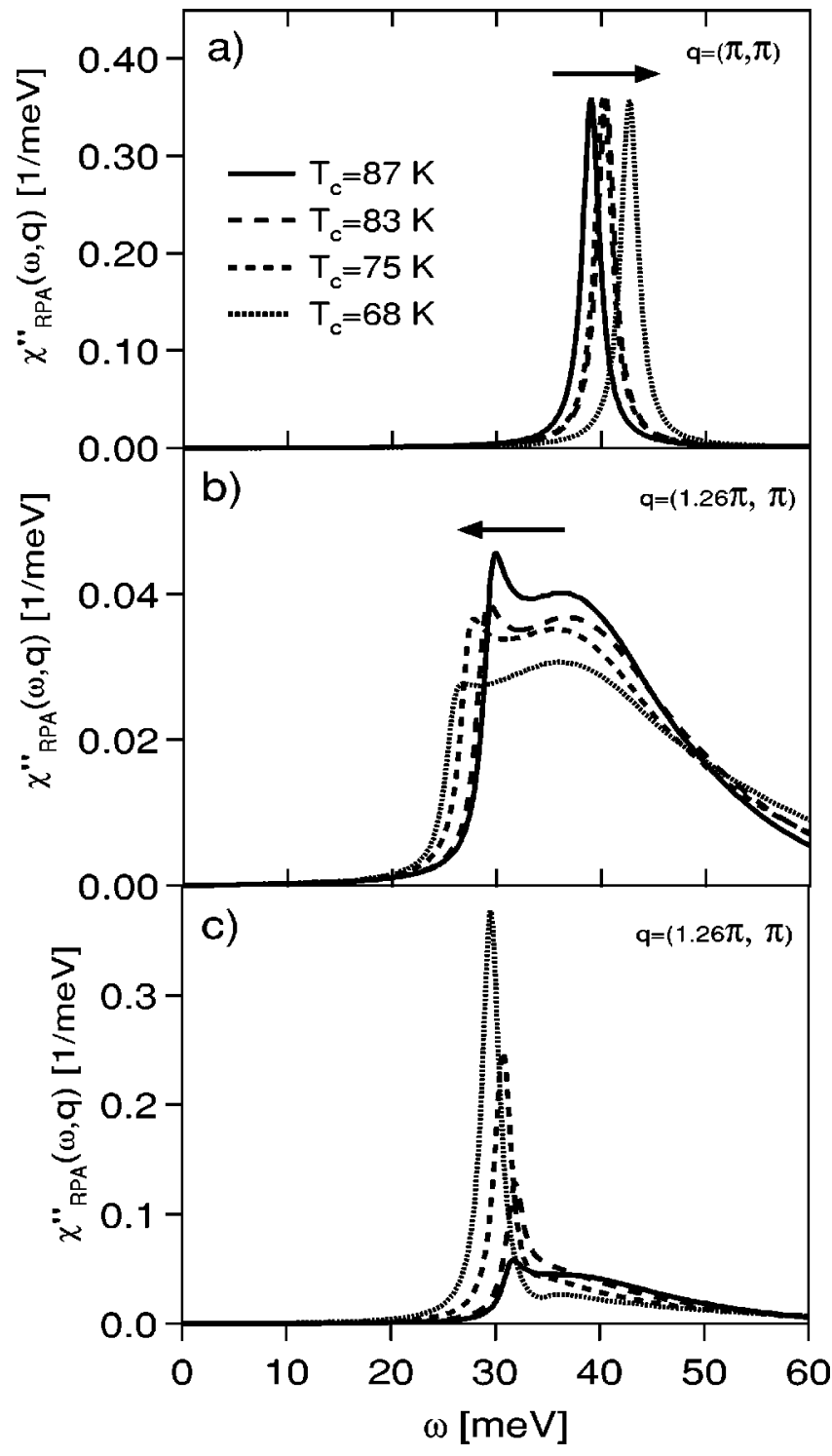

FIG. 6. Panel (a) displays the frequency dependences of $\chi_{\mathrm{RPA}}^{\prime \prime}(\omega, \mathbf{q})$ at the commensurate wave vector $\mathbf{q}=(\pi, \pi)$ for four different values $x$ of doping in the Bi2212 family with the BCS dispersions taken from Table I but with $U$ fixed to $165 \mathrm{meV}$ for all doping concentrations. Panel (b) is the same as panel (a) except for the incommensurate wave vector $\mathbf{q}=(1.26 \pi, \pi)$ being held fixed. Panel (c) displays the frequency dependences of $\chi_{\mathrm{RPA}}^{\prime \prime}(\omega, \mathbf{q})$ at the incommensurate wave vector $\mathbf{q}=(1.26 \pi, \pi)$ for four different values $x$ of doping in the Bi2212 family without inclusion of higher harmonics, i.e., with vanishing $\Delta_{2}$. As explained in the text we take here $\Delta_{1}$ to be $18.8,19.9,20.5$, and $22.1 \mathrm{meV}$, for samples with a $T_{c}$ of $87,83,75$, and $68 \mathrm{~K}$, respectively, and $U$ increases with underdoping from $170 \mathrm{meV}$ at optimal doping to 184,196 , and $211 \mathrm{meV}$ in the underdoped regime. In this way, $U$ is adjusted to reproduce the positions of the resonance as observed in Bi2212. The temperature is here taken to be $T=5 \mathrm{~K}$ while a damping $\Gamma=1 \mathrm{meV}$ is used.

coupled to BCS quasiparticles in the spirit of Refs. 85-89. In both cases we observe a softening of the $\mathbf{q}$-dependent spin gap as a result of switching on higher-order $d$-wave gap harmonics which is rooted in the same mechanism as for the single-band Hubbard model.

\section{CONCLUSIONS}

In conclusion, we have examined the effects of higher $d$-wave gap harmonics induced by underdoping on the dynamical magnetic susceptibility of high- $T_{c}$ cuprates based on a fermiology approach. The calculations are carried out for a single-band Hubbard model with an on-site repulsion treated within the random phase approximation. The input parameters for the BCS dispersions are taken directly from angle resolved photoemission measurements on Bi2212. We find that the inclusion of higher harmonics decreases the q-dependent spin gap to a degree consistent with experiments performed on YBCO. This effect is robust in that it does not depend on the detailed nature of the fermiology model. The downward dispersion of the incommensurate peaks is reproduced and shown to move down in energy with underdoping. We find a crossover from parallel to diagonal incommensuration above the resonance energy. However, this effect depends sensitively on the shape of the BCS dispersions and on the details of the fermiology model, here on the assumption of an on-site Hubbard residual interaction. With the advent of large enough Bi2212 samples for neutron studies it will be possible to compare these predictions to experiments.

Note added. Upon completion of this manuscript, we learned of a related paper by Eremin et al. (Ref. 122) on neutron resonance modes in $d$-wave superconductors, where the same crossover from parallel incommensuration below the resonance energy to diagonal incommensuration above it was found.

\section{ACKNOWLEDGMENTS}

We would like to thank Hiroyuki Yamase and Pengcheng Dai for valuable discussions. This work was supported in parts by the Swiss National Science Foundation under Grant No. 200021-101765/1.

\section{APPENDIX: DEGENERACIES OF $\min _{\mathrm{k}} E_{2}(q, \mathrm{k})$}

The two-particle dispersion $E_{2}(\mathbf{q}, \mathbf{k})$ is $2 \pi$-periodic in all four variables $(\mathbf{k} \in]-\pi, \pi] \times]-\pi, \pi]$ is always in the first Brillouin zone from now on). For any wave vector $\mathbf{q}$, $E_{2}(\mathbf{q}, \mathbf{k})$ is invariant under the involutive symmetry transformation

$$
\left(k_{x}, k_{y}\right) \rightarrow\left(-k_{x}-q_{x},-k_{y}-q_{y}\right),
$$

which can be viewed as a rotation of angle $\pi$ about the point

$$
\left(k_{x}, k_{y}\right)=-\left(\frac{q_{x}}{2}, \frac{q_{y}}{2}\right)
$$

For special values of $\mathbf{q}$ the two-particle dispersion possesses additional symmetries.

At the wave vector $\mathbf{q}=(\pi, \pi), E_{2}$ is left invariant by the point-group transformations of the two-dimensional square lattice. Together with transformation (A1a) the eight elements of the point group form a group of 16 elements. 
(a)

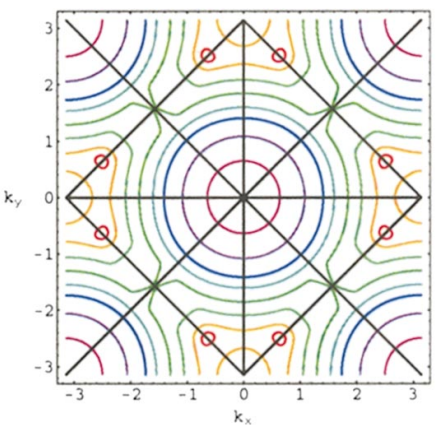

(b)

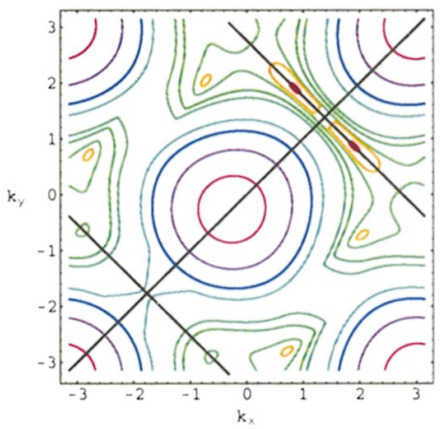

(c)

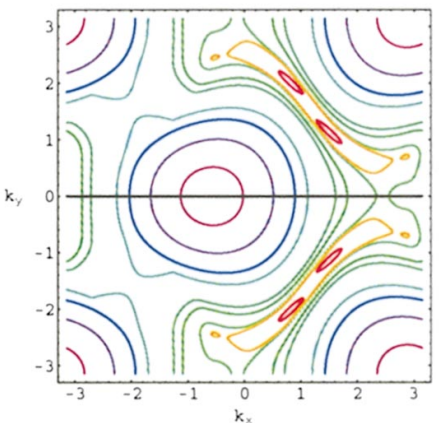

(d)

FIG. 7. (Color) Panel (a) displays the $\mathbf{k}$ dependence of $E_{2}(\mathbf{q}, \mathbf{k})$ at the $\mathbf{q}$ vector $(\pi, \pi)$ in the first Brillouin zone ] $\left.-\pi, \pi\right]$ $\times]-\pi, \pi$ ] for the BCS dispersion of Bi2212 at optimal doping (see Table I). Panels (b), (c), and (d) are the same as panel (a) except for the momentum transfer $\mathbf{q}=(1.1225 \pi, 1.1225 \pi), \mathbf{q}=(1.26 \pi, \pi)$, and $\mathbf{q}=(1.065 \pi, 0.805 \pi)$, respectively. The elevation of the contour lines increases with color, red being the lowest and violet the highest. The axes of reflection are drawn in black. There are one eight-fold degenerate minimum in panel (a), two two-fold degenerate minima and one four-fold degenerate minimum in panel (b), two four-fold degenerate minima in panel (c), and four two-fold degenerate minima in panel (d). These minima are located at the center of the red, orange and green ellipsoidal curves of constant energy. In the discussion we refer to the location of these minima by the q-dependent wave vectors $k_{1}, k_{2}, k_{3}$ and $k_{4}$, respectively.

Hence, the two-dimensional primitive cell in $\mathbf{k}$ space can be divided into 16 triangular subcells which transform into each other under the action of the symmetry group. As the twoparticle energy is a continuous and bounded function in $\mathbf{k}$, $E_{2}(\pi, \pi, \mathbf{k})$ must have at least one minimum and one maximum in each subcell. Furthermore, since the sides of the subcells are mirror axes, the gradient of the two-particle dispersion at $\mathbf{q}=(\pi, \pi)$ vanishes on every corner of the triangles. The degeneracy of the (local) minima of $E_{2}(\pi, \pi, \mathbf{k})$ in the first Brillouin zone is a direct consequence of the symmetry transformations. Depending on whether the minima lie in the interior of the triangles, on the reflection axes, on corners which are shared by four triangles or on corners which are shared by eight triangles, the (local) minima of the two-particle energy are either 16-fold, 8-fold, 4-fold, or 2-fold degenerate, respectively. For the BCS dispersion of Bi2212 at optimal doping (see Table I) the symmetries and the degeneracy of the global minimum of $E_{2}(\pi, \pi, \mathbf{k})$ are depicted in Fig. 7(a).

When $\mathbf{q}$ is chosen to be on either of the two diagonals passing through $(\pi, \pi)$, the two-particle energy is symmetric under the reflection about the corresponding diagonal in the Brillouin zone. Together with transformation (A1a) this yields a symmetry group with four elements. The first Brillouin zone decays into four polygonal subcells which are related to each other by the action of the symmetry group. Hence, the degeneracy of the (local) minima is four-fold, when they are in the interior of the polygons, two-fold, when they lie on a reflection axis and one-fold, when they are on a corner of the subcells. This is illustrated in Fig. 7(b) for the case when $\mathbf{q}$ is on the diagonal connecting $(-\pi, \pi)$ and $(\pi, \pi)$.

If $\mathbf{q}$ is located on the horizontal (vertical) axis passing through the commensurate point $(\pi, \pi), E_{2}$ is left invariant under the reflection about the horizontal (vertical) axis which goes through the origin of the Brillouin zone. The (local) minima are doubly degenerate, when they lie on the reflection axis or on the point (A1b); otherwise they are four-fold degenerate [see Fig. 7(c)].

Finally, when $\mathbf{q}$ is chosen not to be on any of the above symmetry axes, the symmetries of the two-particle energy are reduced to rotation (A1a). The degeneracy of the (local) minima is one-fold, when they lie on the symmetry point (A1b), and two-fold otherwise see [Fig. 7(d)].

From the continuity of $E_{2}(\mathbf{q}, \mathbf{k})$, now considered as a function of $\mathbf{q}$, it follows that the number of local minima of the two-particle energy in the first Brillouin zone for the k's is constant on an open neighborhood of $\mathbf{q}=(\pi, \pi)$. For the BCS dispersions of Table I this neighborhood includes the area around the antiferromagnetic wave vector where most of the spectral weight of $\chi_{\mathrm{RPA}}^{\prime \prime}(\omega, \mathbf{q})$ is located, as is illustrated in Fig. 7. With these hopping and gap parameters the minima of the two-particle energy at the commensurate point $(\pi, \pi)$ are eight-fold degenerate, since they lie on the magnetic Bril- 
louin zone boundary. There is one doubly and one quadruple degenerate local minimum, and a doubly degenerate global minimum, when the momentum transfer lies on a diagonal. If $\mathbf{q}$ is chosen to be on a vertical or horizontal axis passing through the commensurate point $(\pi, \pi)$, the two (local) minima are four-fold degenerate. Finally, when the wave vector $\mathbf{q}$ is not on any of the symmetry axes, there are four doubly degenerate (local) minima.
${ }^{1}$ J. Rossat-Mignod, L. P. Regnault, C. Vettier, P. Bourges, P. Burlet, J. Bossy, J. Y. Henry, and G. Lapertot, Physica C 185, 86 (1991).

${ }^{2}$ H. A. Mook, M. Yethiraj, G. Aeppli, T. E. Mason, and T. Armstrong, Phys. Rev. Lett. 70, 3490 (1993).

${ }^{3}$ B. J. Sternlieb, J. M. Tranquada, G. Shirane, M. Sato, and S. Shamoto, Phys. Rev. B 50, 12915 (1994).

${ }^{4}$ H. F. Fong, B. Keimer, P. W. Anderson, D. Reznik, F. Dogan, and I. A. Aksay, Phys. Rev. Lett. 75, 316 (1995).

${ }^{5}$ P. Dai, M. Yethiraj, H. A. Mook, T. B. Lindemer, and F. Dogan, Phys. Rev. Lett. 77, 5425 (1996).

${ }^{6}$ P. Bourges, L. P. Regnault, Y. Sidis, and C. Vettier, Phys. Rev. B 53, 876 (1996).

${ }^{7}$ H. F. Fong, B. Keimer, D. L. Milius, and I. A. Aksay, Phys. Rev. Lett. 78, 713 (1997).

${ }^{8}$ P. Dai, H. A. Mook, and F. Dogan, Phys. Rev. Lett. 80, 1738 (1998).

${ }^{9}$ H. A. Mook, P. Dai, S. M. Hayden, G. Aeppli, T. G. Perring, and F. Dogan, Nature (London) 395, 580 (1998).

${ }^{10}$ P. Dai, H. A. Mook, S. M. Hayden, G. Aeppli, T. G. Perring, R. D. Hunt, and F. Dogan, Science 284, 1344 (1999).

${ }^{11}$ M. Arai, T. Nishijima, Y. Endoh, T. Egami, S. Tajima, K. Tomimoto, Y. Shiohara, M. Takahashi, A. Garrett, and S. M. Bennington, Phys. Rev. Lett. 83, 608 (1999).

${ }^{12}$ P. Bourges, Y. Sidis, H. F. Fong, L. P. Regnault, J. Bossy, A. Ivanov, and B. Keimer, Science 288, 1234 (2000).

${ }^{13}$ H. F. Fong, P. Bourges, Y. Sidis, L. P. Regnault, J. Bossy, A. Ivanov, D. L. Milius, I. A. Aksay, and B. Keimer, Phys. Rev. B 61, 14773 (2000).

${ }^{14}$ H. A. Mook, P. Dai, F. Doğan, and R. D. Hunt, Nature (London) 404, 729 (2000).

${ }^{15}$ P. Dai, H. A. Mook, R. D. Hunt, and F. Doğan, Phys. Rev. B 63, 054525 (2001).

${ }^{16}$ S. Pailhes, Y. Sidis, P. Bourges, C. Ulrich, V. Hinkov, L. P. Regnault, A. Ivanov, B. Liang, C. T. Lin, C. Bernhard, and B. Keimer, Phys. Rev. Lett. 91, 237002 (2003).

${ }^{17}$ C. Stock, W. J. L. Buyers, R. Liang, D. Peets, Z. Tun, D. Bonn, W. N. Hardy, and R. J. Birgeneau, Phys. Rev. B 69, 014502 (2004).

${ }^{18}$ S. Pailhes, Y. Sidis, P. Bourges, V. Hinkov, A. Ivanov, C. Ulrich, L. P. Regnault, and B. Keimer, cond-mat/0403609 (unpublished).

${ }^{19}$ S. M. Hayden, H. A. Mook, P. Dai, T. G. Perring, and F. Dogan, Nature (London) 429, 531 (2004).

${ }^{20}$ G. Shirane, R. J. Birgeneau, Y. Endoh, P. Gehring, M. A. Kastner, K. Kitazawa, H. Kojima, I. Tanaka, T. R. Thurston, and K. Yamada, Phys. Rev. Lett. 63, 330 (1989).

${ }^{21}$ S. W. Cheong, G. Aeppli, T. E. Mason, H. Mook, S. M. Hayden, P. C. Canfield, Z. Fisk, K. N. Clausen, and J. L. Martinez, Phys. Rev. Lett. 67, 1791 (1991).

${ }^{22}$ T. E. Mason, G. Aeppli, S. M. Hayden, A. P. Ramirez, and H. A.
Mook, Phys. Rev. Lett. 71, 919 (1993).

${ }^{23}$ K. Yamada, S. Wakimoto, G. Shirane, C. H. Lee, M. A. Kastner, S. Hosoya, M. Greven, Y. Endoh, and R. J. Birgeneau, Phys. Rev. Lett. 75, 1626 (1995).

${ }^{24}$ S. M. Hayden, G. Aeppli, H. A. Mook, T. G. Perring, T. E. Mason, S.-W. Cheong, and Z. Fisk, Phys. Rev. Lett. 76, 1344 (1996).

${ }^{25}$ T. E. Mason, A. Schröder, G. Aeppli, H. A. Mook, and S. M. Hayden, Phys. Rev. Lett. 77, 1604 (1996).

${ }^{26}$ G. Aeppli, T. E. Mason, S. M. Hayden, H. A. Mook, and J. Kulda, Science 278, 1432 (1997).

${ }^{27}$ K. Yamada, C. H. Lee, K. Kurahashi, J. Wada, S. Wakimoto, S. Ueki, H. Kimura, Y. Endoh, S. Hosoya, G. Shirane, R. J. Birgeneau, M. Greven, M. A. Kastner, and Y. J. Kim, Phys. Rev. B 57, 6165 (1998).

${ }^{28}$ B. Lake, G. Aeppli, T. E. Mason, A. Schröder, D. F. McMorrow, K. Lefmann, M. Isshiki, M. Nohara, H. Takagi, and S. M. Hayden, Nature (London) 400, 43 (1999).

${ }^{29}$ C. H. Lee, K. Yamada, Y. Endoh, G. Shirane, R. J. Birgeneau, M. A. Kastner, M. Greven, and Y. J. Kim, J. Phys. Soc. Jpn. 69, 1170 (2000).

${ }^{30}$ R. Gilardi, S. Streule, A. Hiess, H. M. Ronnow, M. Oda, N. Momono, M. Ido, and J. Mesot, Physica B 350, 72 (2004).

${ }^{31}$ N. B. Christensen, D. F. McMorrow, H. M. Rønnow, B. Lake, S. M. Hayden, G. Aeppli, T. G. Perring, M. Mangkorntong, M. Nohara, and H. Tagaki, cond-mat/0403439 (unpublished)

${ }^{32}$ H. F. Fong, P. Bourges, Y. Sidis, L. P. Regnault, A. Ivanov, G. D. Gul, N. Koshizuka, and B. Keimer, Nature (London) 398, 588 (1999).

${ }^{33}$ H. A. Mook, F. Dogan, and B. C. Chakoumakos, cond-mat/ 9811100 (unpublished)

${ }^{34}$ J. Mesot, N. Metoki, M. Böhm, A. Hiess, and K. Kadowaki, Physica C 341, 2105 (2000).

${ }^{35}$ H. He, Y. Sidis, P. Bourges, G. D. Gu, A. Ivanov, N. Koshizuka, B. Liang, C. T. Lin, L. P. Regnault, E. Schoenherr, and B. Keimer, Phys. Rev. Lett. 86, 1610 (2001).

${ }^{36}$ H. He, P. Bourges, Y. Sidis, C. Ulrich, L. P. Regnault, S. Pailhes, N. S. Berzigiarova, N. N. Kolesnikov, and B. Keimer, Science 295, 1045 (2002).

${ }^{37}$ C. D. Batista, G. Ortiz, and A. V. Balatsky, Phys. Rev. B 64, 172508 (2001).

${ }^{38}$ J. M. Tranquada, H. Woo, T. G. Perring, H. Goka, G. D. Gu, G. $\mathrm{Xu}, \mathrm{M}$. Fujita, and K. Yamada, Nature (London) 429, 534 (2004).

${ }^{39}$ K. Machida, Physica C 158, 192 (1989).

${ }^{40}$ D. Poilblanc and T. M. Rice, Phys. Rev. B 39, R9749 (1989).

${ }^{41}$ J. Zaanen and O. Gunnarsson, Phys. Rev. B 40, R7391 (1989).

${ }^{42}$ H. J. Schulz, J. Phys. (Paris) 50, 2833 (1989).

${ }^{43}$ V. J. Emery and S. A. Kivelson, Physica C 209, 597 (1993).

${ }^{44}$ For a review, see S. A. Kivelson, I. P. Bindloss, E. Fradkin, V. Oganesyan, J. M. Tranquada, A. Kapitulnik, and C. Howald, 
Rev. Mod. Phys. 75, 1201 (2003).

${ }^{45}$ G. S. Uhrig, K. P. Schmidt, M. Grüninger, cond-mat/0402659 (unpublished).

${ }^{46}$ E. Demler and S. C. Zhang, Phys. Rev. Lett. 75, 4126 (1995).

${ }^{47}$ S. C. Zhang, Science 275, 1089 (1997).

${ }^{48}$ Martin Greiter, Phys. Rev. Lett. 79, 4898 (1997).

${ }^{49}$ E. Demler, H. Kohno, and S.-C. Zhang, Phys. Rev. B 58, 5719 (1998).

${ }^{50}$ J. Brinckmann and P. A. Lee, J. Phys. Chem. Solids 59, 1811 (1998).

${ }^{51}$ O. Tchernyshyov, M. R. Norman, and A. V. Chubukov, Phys. Rev. B 63, 144507 (2001).

${ }^{52}$ Jiang-Ping $\mathrm{Hu}$ and Shou-Cheng Zhang, Phys. Rev. B 64, 100502(R) (2001).

${ }^{53}$ N. Bulut, D. W. Hone, D. J. Scalapino, and N. E. Bickers, Phys. Rev. B 41, 1797 (1990).

${ }^{54}$ H. J. Schulz, Phys. Rev. Lett. 64, 1445 (1990).

${ }^{55}$ N. Bulut and D. J. Scalapino, Phys. Rev. Lett. 68, 706 (1992).

${ }^{56}$ N. Bulut and D. J. Scalapino, Phys. Rev. B 47, 3419 (1993).

${ }^{57}$ P. Bénard, L. Chen, and A.-M. S. Tremblay, Phys. Rev. B 47, 15217 (1993).

${ }^{58}$ M. Lavagna and G. Stemmann, Phys. Rev. B 49, 4235 (1994).

${ }^{59}$ D. van der Marel, Phys. Rev. B 51, 1147 (1995).

${ }^{60}$ N. Bulut and D. J. Scalapino, Phys. Rev. B 53, 5149 (1996).

${ }^{61}$ M. I. Salkola and J. R. Schrieffer, Phys. Rev. B 58, R5944 (1998).

${ }^{62}$ M. R. Norman, Phys. Rev. B 61, 14751 (2000).

${ }^{63}$ M. R. Norman, Phys. Rev. B 63, 092509 (2001).

${ }^{64}$ K. Maki and H. Won, Phys. Rev. Lett. 72, 1758 (1994).

${ }^{65}$ I. I. Mazin and V. M. Yakovenko, Phys. Rev. Lett. 75, 4134 (1995).

${ }^{66}$ G. Blumberg, B. P. Stojkovic, and M. V. Klein, Phys. Rev. B 52, 15741(R) (1995).

${ }^{67}$ J.-X. Li, W.-G. Yin, and C.-D. Gong, Phys. Rev. B 58, 2895 (1998)

${ }^{68}$ T. Tanamoto, K. Kuboki, and H. Fukuyama, J. Phys. Soc. Jpn. 60, 3072 (1991).

${ }^{69}$ T. Tanamoto, H. Kohno, and H. Fukuyama, J. Phys. Soc. Jpn. 62, 717 (1993).

${ }^{70}$ T. Tanamoto, H. Kohno, and H. Fukuyama, J. Phys. Soc. Jpn. 63, 2739 (1994).

${ }^{71}$ G. Stemmann, C. Pépin, and M. Lavagna, Phys. Rev. B 50, 4075 (1994).

${ }^{72}$ J. Brinckmann and P. A. Lee, Phys. Rev. Lett. 82, 2915 (1999).

${ }^{73}$ H. Yamase and H. Kohno, J. Phys. Soc. Jpn. 69, 2151 (2000).

${ }^{74}$ J.-X. Li, C.-Y. Mou, and T. K. Lee, Phys. Rev. B 62, 640 (2000).

${ }^{75}$ H. Yamase and H. Kohno, J. Phys. Soc. Jpn. 70, 2733 (2001).

${ }^{76}$ J. Brinckmann and P. A. Lee, Phys. Rev. B 65, 014502 (2001).

${ }^{77}$ H. Yamase, J. Phys. Soc. Jpn. 71, 1154 (2002).

${ }^{78}$ J.-X. Li and C.-D. Gong, Phys. Rev. B 66, 014506 (2002).

${ }^{79}$ H. Yamase and H. Kohno, Phys. Rev. B 68, 014502 (2003).

${ }^{80}$ J.-X. Li, J. Zhang, and J. Luo, Phys. Rev. B 68, 224503 (2003).

${ }^{81}$ F. Onufrieva and J. Rossat-Mignod, Phys. Rev. B 52, 7572 (1995).

${ }^{82}$ F. Onufrieva, P. Pfeuty, and M. Kiselev, Phys. Rev. Lett. 82, 2370 (1999).

${ }^{83}$ F. Onufrieva and P. Pfeuty, Phys. Rev. B 61, 799 (2000).

${ }^{84}$ F. Onufrieva and P. Pfeuty, Phys. Rev. B 65, 054515 (2002).

${ }^{85}$ D. K. Morr and D. Pines, Phys. Rev. Lett. 81, 1086 (1998).

${ }^{86}$ A. Abanov and A. V. Chubukov, Phys. Rev. Lett. 83, 1652
(1999).

${ }^{87}$ D. K. Morr and D. Pines, Phys. Rev. B 61, R6483 (2000).

${ }^{88}$ D. K. Morr and D. Pines, Phys. Rev. B 62, 15177 (2000).

${ }^{89}$ A. V. Chubukov, B. Janko, and O. Tchernyshyov, Phys. Rev. B 63, 180507(R) (2001).

${ }^{90}$ Q. Si, Y. Zha, K. Levin, and J. P. Lu, Phys. Rev. B 47, 9055 (1993).

${ }^{91}$ Y. Zha, K. Levin, and Q. Si, Phys. Rev. B 47, 9124 (1993).

${ }^{92}$ D. Z. Liu, Y. Zha, and K. Levin, Phys. Rev. Lett. 75, 4130 (1995).

${ }^{93}$ A. J. Millis and H. Monien, Phys. Rev. B 54, 16172 (1996).

${ }^{94}$ Y.-J. Kao, Q. Si, and K. Levin, Phys. Rev. B 61, R11898 (2000).

${ }^{95}$ C.-H. Pao and N. E. Bickers, Phys. Rev. B 51, 16310 (1995).

${ }^{96}$ T. Dahm, D. Manske, and L. Tewordt, Phys. Rev. B 54, 6640 (1996).

${ }^{97}$ T. Takimoto and T. Moriya, J. Phys. Soc. Jpn. 67, 3570 (1998).

${ }^{98}$ T. Dahm, D. Manske, and L. Tewordt, Phys. Rev. B 58, 12454 (1998).

${ }^{99}$ K. Kuroki, R. Arita, and H. Aoki, Phys. Rev. B 60, 9850 (1999).

${ }^{100}$ D. Manske, I. Eremin, and K. H. Bennemann, Phys. Rev. B 63, 054517 (2001).

${ }^{101}$ P. B. Littlewood, J. Zaanen, G. Aeppli, and H. Monien, Phys. Rev. B 48, 487 (1993).

${ }^{102}$ M. Ito, Y. Yasui, S. Iikubo, M. Soda, A. Kobayashi, M. Sato, K. Kakurai, C.-H. Lee, and K. Yamada, J. Phys. Soc. Jpn. 73, 991 (2004).

${ }^{103}$ J. P. Lu, Phys. Rev. Lett. 68, 125 (1992).

${ }^{104}$ H.-Y. Kee and Y. B. Kim, Phys. Rev. B 59, 4470 (1999).

${ }^{105}$ K.-K. Voo, W. C. Wu, J.-X. Li, and T. K. Lee, Phys. Rev. B 61, 9095 (2000).

${ }^{106}$ J. M. Harris, Z.-X. Shen, P. J. White, D. S. Marshall, M. C. Schabel, J. N. Eckstein, and I. Bozovic, Phys. Rev. B 54, R15665 (1996).

${ }^{107}$ J. Mesot, M. R. Norman, H. Ding, M. Randeria, J. C. Campuzano, A. Paramekanti, H. M. Fretwell, A. Kaminski, T. Takeuchi, T. Yokoya, T. Sato, T. Takahashi, T. Mochiku, and K. Kadowaki, Phys. Rev. Lett. 83, 840 (1999).

${ }^{108}$ S. V. Borisenko, A. A. Kordyuk, T. K. Kim, S. Legner, K. A. Nenkov, M. Knupfer, M. S. Golden, J. Fink, H. Berger, and R. Follath, Phys. Rev. B 66, 140509(R) (2002).

${ }^{109}$ T. Eckl, W. Hanke, S. V. Borisenko, A. A. Kordyuk, T. Kim, A. Koitzsch, M. Knupfer, and J. Fink, cond-mat/0402340 (unpublished)

${ }^{110}$ Here, we follow the interpretation from Ref. 107 that the changes in the superconducting gap as measured by ARPES are intrinsic and not caused by disorder effects.

${ }^{111}$ This observation is contradicted by recent thermal conductivity measurements (Ref. 112). In this study the quasiparticle velocity tangential to the Fermi surface has been extracted in an indirect way by fitting the data with a simple model. However, APRES experiments provide a direct measurement of the gap dispersion, and the decrease of the gap slope with underdoping has now been establish in several independent experiments (Ref. 106-109). These findings are further supported by lowtemperature penetration depth measurements (Ref. 113).

${ }^{112}$ M. Sutherland, D. G. Hawthorn, R. W. Hill, F. Ronning, S. Wakimoto, H. Zhang, C. Proust, E. Boaknin, C. Lupien, L. Taillefer, R. Liang, D. A. Bonn, W. N. Hardy, R. Gagnon, N. E. Hussey, T. Kimura, M. Nohara, and H. Takagi, Phys. Rev. B 67, 174520 (2003). 
${ }^{113}$ Ch. Panagopoulos and T. Xiang, Phys. Rev. Lett. 81, 2336 (1998).

${ }^{114}$ The YBCO and Bi2212 families are stacked bilayers. One expects that the quasiparticle dispersions close to the Fermi energy split into bonding and antibonding bands. This was first seen in both ARPES and neutron scattering on YBCO. [Refs. 13 and 16; see also M. C. Schabel, C.-H. Park, A. Matsuura, Z.-X. Shen, D. A. Bonn, X. Liang, and W. N. Hardy, Phys. Rev. B 57, 6090 (1998).]. However, in the underdoped and optimally doped regimes the prominent incommensurate and commensurate peaks in the magnetic susceptibility are only observed in the antibonding band. Peaks are observed in the bonding band but they are very broad both as a function of energy and as a function of momentum. Recently a resonant excitation in the even channel was found for overdoped YBCO, albeit with a much smaller intensity than the odd channel resonance (Ref. 16). It seems most likely that the resonance in the bonding band is only present in the overdoped regime and grows in intensity as one increases the doping concentration. Within the fermiology approach it was shown in Ref. 76 (see also Refs. 60, 65, 66, 92, 93, and 96) that an effective one-band model for the antibonding band is sufficient to account for the incommensurate and commensurate peaks in the YBCO and Bi2212 families, if one only considers optimally doped and underdoped samples. We shall adopt the same point of view in this paper.

${ }^{115}$ A. V. Chubukov and M. R. Norman, cond-mat/0402304 (unpublished).

${ }^{116}$ M. R. Norman, M. Randeria, H. Ding, and J. C. Campuzano, Phys. Rev. B 52, 615 (1995).

${ }^{117}$ H. Ding, M. R. Norman, T. Yokoya, T. Takeuchi, M. Randeria, J. C. Campuzano, T. Takahashi, T. Mochiku, and K. Kadowaki, Phys. Rev. Lett. 78, 2628 (1997).

${ }^{118}$ A. A. Kordyuk, S. V. Borisenko, M. S. Golden, S. Legner, K. A. Nenkov, M. Knupfer, J. Fink, H. Berger, L. Forro, and R. Follath, Phys. Rev. B 66, 014502 (2002).

${ }^{119}$ J. C. Campuzano, H. Ding, M. R. Norman, H. M. Fretwell, M. Randeria, A. Kaminski, J. Mesot, T. Takeuchi, T. Sato, T. Yokoya, T. Takahashi, T. Mochiku, K. Kadowaki, P. Guptasarma, D. G. Hinks, Z. Konstantinovic, Z. Z. Li, and H. Raffy, Phys. Rev. Lett. 83, 3709 (1999).

${ }^{120}$ J. F. Zasadzinski, L. Ozyuzer, N. Miyakawa, K. E. Gray, D. G. Hinks, and C. Kendziora, Phys. Rev. Lett. 87, 067005 (2001).

${ }^{121}$ The coordinate system $\left(k_{1}, k_{2}\right)$ is defined by demanding that 0 $=\partial^{2} E_{2}(\mathbf{q}, \mathbf{k}) / \partial k_{1} \partial k_{2}$ when $\mathbf{k}=\mathbf{k}_{n}$

${ }^{122}$ I. Eremin, D. K. Morr, A. V. Chubukov, K. Bennemann, and M. R. Norman, cond-mat/0409599 (unpublished). 